\title{
A family of reductions for Schubert intersection problems
}

\author{
H. Bercovici · W.S. Li $\cdot$ D. Timotin
}

Received: 4 September 2009 / Accepted: 18 October 2010 / Published online: 6 November 2010

(C) Springer Science+Business Media, LLC 2010

\begin{abstract}
We produce a family of reductions for Schubert intersection problems whose applicability is checked by calculating a linear combination of the dimensions involved. These reductions do not alter the Littlewood-Richardson coefficient, and this fact is connected to known multiplicative properties of these coefficients.
\end{abstract}

Keywords Schubert variety $\cdot$ Littlewood-Richardson rule $\cdot$ Puzzle $\cdot$ Tree $\cdot$ Measure

\section{Introduction}

Given integers $n>r \geq 1$, we denote by $G\left(r, \mathbb{C}^{n}\right)$ the Grassmannian manifold consisting of all $r$-dimensional subspaces in $\mathbb{C}^{n}$. For every flag

$$
\mathcal{E}=\left\{\{0\}=\mathbb{E}_{0} \subset \mathbb{E}_{1} \subset \mathbb{E}_{2} \subset \cdots \subset \mathbb{E}_{n}=\mathbb{C}^{n}\right\},
$$

where $\mathbb{E}_{j}$ is a subspace of dimension $j, G\left(r, \mathbb{C}^{n}\right)$ can be written as a union of Schubert varieties described as follows. For each set $I=\left\{i_{1}<i_{2}<\cdots<i_{r}\right\} \subset$

H.B. and W.S.L. were supported in part by grants from the National Science Foundation.

H. Bercovici $(\bowtie)$

Department of Mathematics, Indiana University, Bloomington, IN 47405, USA

e-mail: bercovic@indiana.edu

W.S. Li

School of Mathematics, Georgia Institute of Technology, Atlanta, GA 30332-1060, USA

e-mail: li@math.gatech.edu

D. Timotin

Simion Stoilow Institute of Mathematics of the Romanian Academy, PO Box 1-764, Bucharest

014700, Romania

e-mail: Dan.Timotin@imar.ro 
$\{1,2, \ldots, n\}$ one defines the Schubert variety

$$
\mathfrak{S}(\mathcal{E}, I)=\left\{\mathbb{M} \in G\left(r, \mathbb{C}^{n}\right): \operatorname{dim}\left(\mathbb{M} \cap \mathbb{E}_{i_{x}}\right) \geq x, x=1,2, \ldots, r\right\} .
$$

Schubert calculus allows one to find the number of points in the intersection of several Schubert varieties $\mathfrak{S}\left(\mathcal{E}_{\ell}, I_{\ell}\right), \ell=1,2, \ldots, s$, when the flags $\left(\mathcal{E}_{\ell}\right)_{\ell=1}^{s}$ are in generic position. We will be mostly concerned with the case $s=3$ where the classical Littlewood-Richardson rule applies (cf. [6]). Thus, given sets $I, J, K \subset\{1,2, \ldots, n\}$ of cardinality $r$ such that

$$
\sum_{\ell=1}^{r}\left(i_{\ell}+j_{\ell}+k_{\ell}-3 \ell\right)=2 r(n-r),
$$

the Littlewood-Richardson rule (which will be reviewed below) provides a nonnegative integer $c_{I J K}$ with the property that the set

$$
S=\mathfrak{S}(\mathcal{E}, I) \cap \mathfrak{S}(\mathcal{F}, J) \cap \mathfrak{S}(\mathcal{G}, K)
$$

contains $c_{I J K}$ elements for generic flags $\mathcal{E}, \mathcal{F}, \mathcal{G}$. For non-generic flags, this intersection is still certain to be nonempty if $c_{I J K}>0$.

Thompson and Therianos [9] pointed out that under certain circumstances one can reduce the problem of finding elements in the set $S$ to a problem where $n$ is replaced by a smaller number. In order to explain their reductions, it will be convenient to set $I=\left\{i_{1}<i_{2}<\cdots<i_{r}\right\}$, define $i_{0}=0$, and similarly $j_{0}=k_{0}=0$. Assume that the indices $x, y, z \in\{0,1,2, \ldots, r\}$ are such that $x+y+z=r$ and $i_{x}+j_{y}+k_{z}=$ $n-p<n$. In this case, the spaces $\mathbb{E}_{i_{x}}, \mathbb{F}_{j_{y}}, \mathbb{G}_{k_{z}}$ are generically independent, and for any space $\mathbb{M} \in S$ we have

$$
r=\operatorname{dim}(\mathbb{M}) \geq \operatorname{dim}\left(\mathbb{M} \cap \mathbb{E}_{i_{x}}\right)+\operatorname{dim}\left(\mathbb{M} \cap \mathbb{F}_{j_{y}}\right)+\operatorname{dim}\left(\mathbb{M} \cap \mathbb{G}_{k_{z}}\right) \geq x+y+z=r .
$$

Therefore $\mathbb{M}$ is contained in $\mathbb{E}_{i_{x}}+\mathbb{F}_{j_{y}}+\mathbb{G}_{k_{z}}$. Replace now $\mathbb{C}^{n}$ by the space $\mathbb{X}=$ $\mathbb{E}_{i_{x}}+\mathbb{F}_{j_{y}}+\mathbb{G}_{k_{z}}$ and the spaces $\mathbb{E}_{i}, \mathbb{F}_{j}, \mathbb{G}_{k}$ by their intersections with $\mathbb{X}$. Observe that generically

$$
\operatorname{dim}\left(\mathbb{E}_{i} \cap \mathbb{X}\right)= \begin{cases}i & \text { if } i \leq i_{x} \\ i_{x} & \text { if } i_{x}<i \leq i_{x}+p \\ i-p & \text { if } i_{x}+p<i \leq n\end{cases}
$$

and these spaces will form (after the repeating spaces of dimension $i_{x}$ are deleted) a flag $\mathcal{E}^{\prime}$ in $\mathbb{X}$. Flags $\mathcal{F}^{\prime}$ and $\mathcal{G}^{\prime}$ are defined similarly. Finding the spaces in $S$ amounts to finding the spaces in

$$
S^{\prime}=\mathfrak{S}\left(\mathcal{E}^{\prime}, I^{\prime}\right) \cap \mathfrak{S}\left(\mathcal{F}^{\prime}, J^{\prime}\right) \cap \mathfrak{S}\left(\mathcal{G}^{\prime}, K^{\prime}\right) \subset G(r, \mathbb{X}),
$$

where

$$
i_{\ell}^{\prime}= \begin{cases}i_{\ell} & \text { if } 1 \leq \ell \leq x, \\ i_{\ell}-p & \text { if } x<\ell \leq r\end{cases}
$$


with similar definitions for $J^{\prime}$ and $K^{\prime}$. (The sequence $\left(i_{\ell}^{\prime}\right)_{\ell=1}^{r}$ is still strictly increasing because the condition $i_{x}+j_{y}+k_{z}=n-p$ actually implies that $i_{x+1}>i_{x}+p$ provided that $c_{I J K}>0$.) The question arises naturally whether $c_{I^{\prime} J^{\prime} K^{\prime}} \neq 0$ if $c_{I J K} \neq 0$, so that the reduced problem is still guaranteed to have a solution. That this is indeed the case was shown by Collins and Dykema [3] who proved that in fact $c_{I^{\prime} J^{\prime} K^{\prime}}=c_{I J K}$.

The purpose of this paper is to identify a much larger family of reductions associated with various inequalities satisfied by $I, J, K$. This family is sufficient for the complete solution of the intersection problem when $c_{I J K}=1$. The simplest of these new reductions is as follows. Assume that $c_{I J K}>0, x, y, z \in\{1,2, \ldots, r\}$ satisfy $x+y+z=2 r$, and

$$
i_{x}+j_{y}+k_{z}=2 n-p<2 n
$$

In this case the space

$$
\mathbb{X}=\left(\mathbb{E}_{i_{x}} \cap \mathbb{F}_{j_{y}}\right)+\left(\mathbb{E}_{i_{x}} \cap \mathbb{G}_{k_{z}}\right)+\left(\mathbb{F}_{j_{y}} \cap \mathbb{G}_{k_{z}}\right)
$$

has generically codimension $2 p$ and it contains all the spaces in $S$. The reduced problem in $G(r, \mathbb{X})$ corresponds with the sets $I^{\prime}, J^{\prime}, K^{\prime}$ defined by

$$
i_{\ell}^{\prime}= \begin{cases}i_{\ell}-p & \text { if } 1 \leq \ell \leq i_{x}, \\ i_{\ell}-2 p & \text { if } i_{x}<\ell \leq r\end{cases}
$$

with analogous definitions for $j_{\ell}^{\prime}, k_{\ell}^{\prime}$. As in the result of [3] just mentioned, we have $c_{I^{\prime} J^{\prime} K^{\prime}}=c_{I J K}$. The general reduction we propose can be described as follows. We are given $r$-tuples $a=\left(a_{\ell}\right)_{\ell=1}^{r}, b=\left(b_{\ell}\right)_{\ell=1}^{r}, c=\left(c_{\ell}\right)_{\ell=1}^{r}$ of non-negative integers such that

$$
\sum_{\ell=1}^{r}\left(\ell a_{\ell}+\ell b_{\ell}+\ell c_{\ell}\right)=\omega r
$$

for some positive integer $\omega ; a, b, c$ are subject to other conditions which will be discussed later. Assume that the sets $I, J, K \subset\{1,2, \ldots, n\}$ have cardinality $r$, $c_{I J K}>0$, and consider the sum

$$
\sum_{\ell=1}^{r}\left(a_{\ell} i_{\ell}+b_{\ell} j_{\ell}+c_{\ell} k_{\ell}\right)=\omega n-p,
$$

where $p$ is some integer. The reduction corresponding to $a, b, c$ can be applied when $p>0$. Namely, if $p>0$, we necessarily have $\omega p \leq n$. Moreover, there exist

(1) a space $\mathbb{X} \subset \mathbb{C}^{n}$ with $\operatorname{dim} \mathbb{X}=n-\omega p$,

(2) flags $\mathcal{E}^{\prime}, \mathcal{F}^{\prime}, \mathcal{G}^{\prime}$ in $\mathbb{X}$,

(3) sets $I^{\prime}, J^{\prime}, K^{\prime} \subset\{1,2, \ldots, n-\omega p\}$ of cardinality $r$ such that $c_{I^{\prime} J^{\prime} K^{\prime}}=c_{I J K}$ and

$$
\mathfrak{S}\left(\mathcal{E}^{\prime}, I^{\prime}\right) \cap \mathfrak{S}\left(\mathcal{F}^{\prime}, J^{\prime}\right) \cap \mathfrak{S}\left(\mathcal{G}^{\prime}, K^{\prime}\right) \subset \mathfrak{S}(\mathcal{E}, I) \cap \mathfrak{S}(\mathcal{F}, J) \cap \mathfrak{S}(\mathcal{G}, K) .
$$


In addition, the space $\mathbb{X}$ can be constructed (when the flags $\mathcal{E}, \mathcal{F}, \mathcal{G}$ are in 'general position') explicitly from $\mathcal{E}, \mathcal{F}, \mathcal{G}$ by applying a finite number of sums and intersections. The sequences $a, b, c$ which appear here are themselves related to the LittlewoodRichardson rule.

The two reductions discussed above are such that the only nonzero components of $a, b, c$ are $a_{x}=b_{y}=c_{z}=1$, and $\omega=1$ or $\omega=2$.

Our proofs deepen some of the results in [1]. Even though we review the relevant results of [1], familiarity with that paper would be helpful in reading this one. The paper benefited from a careful reading by the referees who helped improve the exposition, and suggested that this work is connected to earlier results about the factorization of Littlewood-Richardson coefficients [5, 7]. The results in Sect. 7 arose from this suggestion. The second part of Theorem 3.1 also answers a natural question raised by one of the referees.

The remainder of the paper is organized as follows. In Sect. 2 we describe the formulation of the Littlewood-Richardson rule in terms of measures. This is essentially the puzzle formulation of [8], and was also used in [1]. We also introduce the linear combinations of dimensions which serve as witnesses for the possibility of reductions. In Sect. 3 we discuss a special class of measures, the tree measures. It was implicit in the results of [1] that rigid extremal measures have an underlying tree structure, and this is made explicit here. Section 4 reviews the construction of a puzzle from a measure, and uses the results of Sect. 3 to deduce the identity $c_{I^{\prime} J^{\prime} K^{\prime}}=c_{I J K}$. In Sect. 5 we prove the essential technical result needed to show in Sect. 6 that the analogues of the reductions of [9] can indeed be performed. In Sect. 7 we relate our results to previous work [5, 7] on the factorization of Littlewood-Richardson coefficients. In particular, after applying an appropriate duality, Corollary 4.3 is seen as a consequence of factorization results. It seems practically impossible to describe all rigid tree measures in a uniform manner, and an exhaustive application of our reductions does require knowledge of these tree measures. We provide in Sect. 8 a description of a fairly large class of such measures.

\section{The Littlewood-Richardson rule}

We now give the description of the Littlewood-Richardson rule in terms of measures. This is equivalent with the puzzle description of [8]. Choose unit vectors $w_{1}, w_{2}, w_{3}$ in the plane such that $w_{1}+w_{2}+w_{3}=0$.

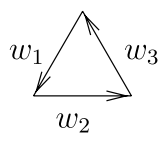

The points $i w_{1}+j w_{2}$ with integer $i, j$ will be called lattice points, and a segment joining two nearest lattice points will be called a small edge. We consider positive measures $m$ which are supported by the union of the small edges, whose restriction to each small edge is a multiple of arclength measure, and which satisfy the balance condition (called zero tension in [8])

$$
m(A B)-m\left(A B^{\prime}\right)=m(A C)-m\left(A C^{\prime}\right)=m(A D)-m\left(A D^{\prime}\right)
$$


whenever $A$ is a lattice point and the neighboring lattice points $B, C^{\prime}, D, B^{\prime}, C, D^{\prime}$ are in cyclic order around $A$.

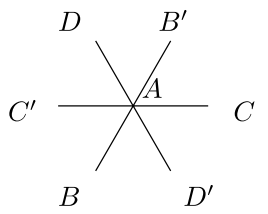

If $e$ is a small edge, the value $m(e)$ is equal to the density of $m$ relative to arclength measure on that edge.

Fix now an integer $r \geq 1$, and denote by $\triangle_{r}$ the (closed) triangle with vertices $0, r w_{1}$, and $r w_{1}+r w_{2}=-r w_{3}$. We will use the notation $A_{j}=j w_{1}, B_{j}=r w_{1}+$ $j w_{2}$, and $C_{j}=(r-j) w_{3}$ for the lattice points on the boundary of $\triangle_{r}$. We also set

$$
X_{j}=A_{j}+w_{3}, \quad Y_{j}=B_{j}+w_{1}, \quad Z_{j}=C_{j}+w_{2}
$$

for $j=0,1,2, \ldots, r+1$. The following picture represents $\triangle_{5}$ and the points just defined; the labels are placed on the left.

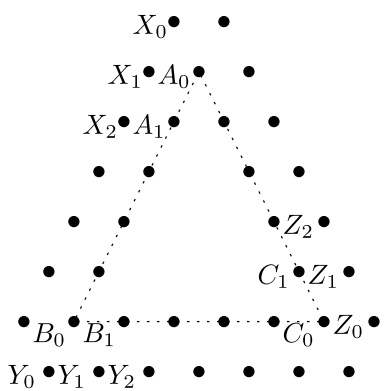

Given a measure $m$, a branch point is a lattice point incident to at least three edges in the support of $m$. We only consider measures with at least one branch point. This excludes measures whose support consists of one or more parallel lines. We denote by $\mathcal{M}_{r}$ the collection of all measures $m$ satisfying the balance condition above, whose branch points are contained in $\triangle_{r}$, and such that

$$
m\left(A_{j} X_{j+1}\right)=m\left(B_{j} Y_{j+1}\right)=m\left(C_{j} Z_{j+1}\right)=0, \quad j=0,1, \ldots, r .
$$

The numbers $\alpha_{j}=m\left(A_{j} X_{j}\right), \beta_{j}=m\left(B_{j} Y_{j}\right)$ and $\gamma_{j}=m\left(C_{j} Z_{j}\right)$ are called the exit densities of $m$. The weight $\omega(m)$ of a measure $m \in \mathcal{M}_{r}$ is defined as

$$
\omega(m)=\sum_{j=0}^{r} \alpha_{j}=\sum_{j=0}^{r} \beta_{j}=\sum_{j=0}^{r} \gamma_{j}
$$

the equality of the three sums follows from the balance condition.

In an analogous fashion, we denote by $\mathcal{M}_{r}^{*}$ the collection of all measures $m$ satisfying the balance condition (2.1), whose branch points are contained in $\triangle_{r}$, and such 
that

$$
m\left(A_{j} X_{j}\right)=m\left(B_{j} Y_{j}\right)=m\left(C_{j} Z_{j}\right)=0, \quad j=0,1, \ldots, r .
$$

The exit densities of such a measure are $\alpha_{r-j}=m\left(A_{j} X_{j+1}\right), \beta_{r-j}=m\left(B_{j} Y_{j+1}\right)$, and $\gamma_{r-j}=m\left(C_{j} Z_{j+1}\right)$ for $j=0,1, \ldots, r$, and the weight $\omega(m)$ is defined as in the case of $\mathcal{M}_{r}$.

Assume that $m \in \mathcal{M}_{r}$ assigns integer densities to all small edges. We can then define an integer

$$
n=r+\omega(m)
$$

and sets $I, J, K \subset\{1,2, \ldots, n\}$ of cardinality $r$ by setting $I=\left\{i_{1}, i_{2}, \ldots, i_{r}\right\}$, where

$$
i_{\ell}=\ell+\sum_{j=0}^{\ell-1} \alpha_{j}, \quad \ell=1,2, \ldots, r
$$

with similar formulas for $J$ and $K$. These are precisely the triples of sets $(I, J, K)$ which satisfy the Littlewood-Richardson rule. Given sets or $r$ elements $I, J, K \subset$ $\{1,2, \ldots, n\}$, the Littlewood-Richardson coefficient $c_{I J K}$ equals the number of measures $m \in \mathcal{M}_{r}$ with integer densities which satisfy $\omega(m)=n-r$ and (2.2) and its analogues for $J$ and $K$. (See [8], or [2, Appendix] for a direct proof of this fact.) We also write $c_{m}=c_{I J K}$ when $I, J, K$ are obtained from $m$. When $c_{m}=1$, we say that $m$ is rigid. In other words, $m$ is rigid if there is no other measure with the same exit densities. Note that knowledge of $n$ and of the sets $I, J, K$ determines entirely the numbers $\alpha_{j}, \beta_{j}, \gamma_{j}$. Thus the Littlewood-Richardson rule states, in particular, that $c_{I J K}>0$ if and only if these numbers are actual exit densities of some measure.

One of the advantages of this formulation of the Littlewood-Richardson rule is that it displays an underlying convexity structure. Thus, the set $\mathcal{M}_{r}$ is a convex polyhedral cone, and therefore each measure $0 \neq m \in \mathcal{M}_{r}$ can be written as a sum of extremal measures. Recall that $m \neq 0$ is extremal if every measure $m^{\prime} \leq m$ is a multiple of $m$. This decomposition into extremal summands is unique (except for the order of the terms) if $m$ is a rigid measure (see [1, Corollary 3.6]). In the proof of Theorem 3.1, we will describe briefly the result of [1] showing how the extremal summands of a rigid measure are obtained.

The results of [8] imply that, given a measure $m \in \mathcal{M}_{r}$ with exit densities $\alpha_{j}, \beta_{j}, \gamma_{j}$, there exist Hermitian $r \times r$ matrices $X, Y, Z$ such that $X+Y+Z=$ $2 \omega(m) 1_{r}$, and the eigenvalues of $X, Y, Z$ are, respectively, the numbers

$$
\sum_{j=0}^{\ell-1} \alpha_{j}, \quad \sum_{j=0}^{\ell-1} \beta_{j}, \quad \sum_{j=0}^{\ell-1} \gamma_{j}, \quad \ell=1,2, \ldots, r ;
$$

here $1_{r}$ denotes the $r \times r$ identity matrix. The sum of the traces of $X, Y, Z$ must then be $2 r \omega(m)$, and this can be written in the equivalent form

$$
\sum_{\ell=0}^{r} \ell\left(\alpha_{\ell}+\beta_{\ell}+\gamma_{\ell}\right)=r \omega(m)
$$


As seen in the Introduction, the possibility of reductions for the Schubert intersection problem defined by the sets $I, J, K \subset\{1,2, \ldots, n\}$ is tested by calculating an appropriate sum of the indices in these sets. We are now ready to discuss these sums in full generality. Assume therefore that $r$ is fixed, $I, J, K \subset\{1,2, \ldots, n\}$ and $I^{\prime}, J^{\prime}, K^{\prime} \subset\left\{1,2, \ldots, n^{\prime}\right\}$ are sets of cardinality $r$ such that $c_{I J K}>0$ and $c_{I^{\prime} J^{\prime} K^{\prime}}>0$. Let us set $\omega=n-r$ and $\omega^{\prime}=n^{\prime}-r$. Choose measures $m, m^{\prime} \in \mathcal{M}_{r}$ such that $\omega(m)=\omega, \omega\left(m^{\prime}\right)=\omega^{\prime}$, and $I, J, K$ (resp. $\left.I^{\prime}, J^{\prime}, K^{\prime}\right)$ are derived from $m$ (resp. $\left.m^{\prime}\right)$ via (2.2). We denote by $\alpha_{\ell}, \beta_{\ell}, \gamma_{\ell}$ (resp. $\alpha_{\ell}^{\prime}, \beta_{\ell}^{\prime}, \gamma_{\ell}^{\prime}$ ) the exit densities of $m$ (resp. $m^{\prime}$ ). The sum we are interested in is

$$
\Sigma_{m^{\prime}}(m)=\sum_{\ell=1}^{r}\left(\alpha_{\ell}^{\prime} i_{\ell}+\beta_{\ell}^{\prime} j_{\ell}+\gamma_{\ell}^{\prime} k_{\ell}\right)-\omega^{\prime} n .
$$

Observe that $\Sigma_{m^{\prime}}(m)$ depends only on the exit densities of $m$ and $m^{\prime}$, and therefore it can be calculated directly from the sets $I, J, K$ and $I^{\prime}, J^{\prime}, K^{\prime}$.

The general reduction will proceed as follows. Assume that we want to solve the Schubert problem associated to a measure $m \in \mathcal{M}_{r}$. We calculate the sum $\Sigma_{m^{\prime}}(m)$ for a certain kind of measure $m^{\prime}$ (a rigid tree measure in the terminology introduced below). If this sum is equal to $-p<0$, then $p m^{\prime} \leq m$ and one can effectively reduce the intersection problem to solving first an intersection problem for a stretched version of $m^{\prime}$, followed by the intersection problem for $m-p m^{\prime}$, for which we have $c_{m-p m^{\prime}}=c_{m}$; see Theorem 6.2. The problem corresponding to the stretched version of $m^{\prime}$ can be solved algorithmically, as seen in [1].

Since $n=\omega(m)+r$, we can rewrite

$$
\begin{aligned}
\Sigma_{m^{\prime}}(m)= & \sum_{\ell<\ell^{\prime}}\left(\alpha_{\ell} \alpha_{\ell^{\prime}}^{\prime}+\beta_{\ell} \beta_{\ell^{\prime}}^{\prime}+\gamma_{\ell} \gamma_{\ell^{\prime}}^{\prime}\right)-\omega(m) \omega\left(m^{\prime}\right) \\
& +\left[\sum_{\ell=0}^{r} \ell\left(\alpha_{\ell}^{\prime}+\beta_{\ell}^{\prime}+\gamma_{\ell}^{\prime}\right)-r \omega\left(m^{\prime}\right)\right] .
\end{aligned}
$$

We have seen earlier that the sum inside the brackets is equal to zero, and thus

$$
\Sigma_{m^{\prime}}(m)=\sum_{\ell<\ell^{\prime}}\left(\alpha_{\ell} \alpha_{\ell^{\prime}}^{\prime}+\beta_{\ell} \beta_{\ell^{\prime}}^{\prime}+\gamma_{\ell} \gamma_{\ell^{\prime}}^{\prime}\right)-\omega(m) \omega\left(m^{\prime}\right)
$$

This formula has several advantages: it does not depend explicitly on $r$, and by including the branch points of $m$ and $m^{\prime}$ in a triangle of a different size we do not alter the sum. More precisely, if we enlarge the triangle containing the branch points of the measures, the value of $r$ changes, but the nonzero values $\alpha_{\ell}, \alpha_{\ell}^{\prime}$ remain the same, and they appear in the same order, leaving the sum $\Sigma_{m^{\prime}}(m)$ unchanged. The arguments in the remainder of the paper are easier to visualize when all the branch points are contained in the interior of $\triangle_{r}$, and the reader is free to make this additional assumption at any point. Another change which does not affect the value of $\Sigma_{m^{\prime}}(m)$ is homothety. Denote by $S$ and $S^{\prime}$ the supports of $m$ and $m^{\prime}$, and let $q$ be a positive integer. It is then possible to define measures $\mu$ and $\mu^{\prime}$ supported by $q S$ and $q S^{\prime}$, respectively, and such that the density of each segment of the form qe equals the original density of $e$. It 
is obvious that $\Sigma_{\mu^{\prime}}(\mu)=\Sigma_{m^{\prime}}(m)$. Taking, for instance, $q=2$, each small edge in the support of $m$ turns into two collinear small edges in the support of $\mu$. It is thus possible to assume that for every small edge $e$ in the support of $m$ there is a second, collinear, edge $e^{\prime}$ which meets $e$ in a vertex $V$ which is not a branch point. This is a formal way to perform an operation which is referred to as 'breaking an edge in half' later on.

The fact that $\omega(m) \omega\left(m^{\prime}\right)=\sum_{\ell, \ell^{\prime}=1}^{r} \alpha_{\ell} \alpha_{\ell^{\prime}}^{\prime}$ implies easily that

$$
\Sigma_{m^{\prime}}(m)+\Sigma_{m}\left(m^{\prime}\right)=\omega(m) \omega\left(m^{\prime}\right)-\sum_{\ell=1}^{r}\left(\alpha_{\ell} \alpha_{\ell}^{\prime}+\beta_{\ell} \beta_{\ell}^{\prime}+\gamma_{\ell} \gamma_{\ell}^{\prime}\right) .
$$

In particular, when $m=m^{\prime}$ we have

$$
\Sigma_{m}(m)=\frac{1}{2}\left[\omega(m)^{2}-\sum_{\ell=1}^{r}\left(\alpha_{\ell}^{2}+\beta_{\ell}^{2}+\gamma_{\ell}^{2}\right)\right]
$$

a formula requiring fewer multiplications.

\section{Trees and measures}

Some measures $m \in \mathcal{M}_{r}$ have an underlying tree structure which we describe next. We start with a special class of trees. We consider labeled trees such that

(1) each edge is assigned a label from the set $\{1,2,3\}$ called the type of that edge,

(2) each vertex has order 2 or 3 ,

(3) the two edges adjacent to a vertex of order 2 have the same label,

(4) the three edges adjacent to a vertex of order 3 have distinct labels, and

(5) there are only finitely many vertices of order 3.

These conditions imply that the tree is infinite, but it has a finite number of ends. These are sequences of vertices of the form $V_{0} V_{1} \cdots$ such that $V_{0}$ has order $3, V_{j}$ has order 2 for $j \geq 1$, and $V_{j} V_{j+1}$ is an edge for each $j \geq 0$. We will impose one more condition on our trees.

(6) The shortest path joining two different ends contains an odd number of vertices of order 3 .

All the trees we use will satisfy these four properties, and therefore we will not introduce a special name for this particular species.

An immersion of a tree $T \subset \mathbb{R}^{2}$ is simply a continuous map $\varphi: T \rightarrow \mathbb{R}^{2}$ which

(i) maps each edge of type $j \in\{1,2,3\}$ homeomorphically onto a small edge parallel to $w_{j}$,

(ii) if $V A$ and $V B$ are the two edges meeting at a vertex of order 2 , then $2 \varphi(V)=$ $\varphi(A)+\varphi(B)$, and 
(iii) if $V A, V B, V C$ are the three edges meeting at a vertex of order 3 , then $3 \varphi(V)=$ $\varphi(A)+\varphi(B)+\varphi(C)$.

Condition (iii) actually follows from (i). If we endow $T$ with a metric such that each edge is isometric to the segment $[0,1]$, and require that immersions are isometric on each edge, then $T$ has a unique immersion up to translations and symmetries with respect to lattice points. It is easy to see that each of the trees we consider can be embedded into $\mathbb{R}^{2}$ in such a way that each edge is identified with a straight line segment of unit length. In the following illustrations we always embed trees that way, and consider only immersions which preserve the orientation of the edges around each vertex of order 3 . With this convention, the types of all edges are determined by the type of one edge.

Immersions are generally not one-to-one.

Assume now that $T$ is metrized as above, and endow it with arclength measure. Given an immersion $\varphi$ of $T$ which is isometric on each edge, we consider the pushforward $m_{\varphi}$ of this measure. Thus $m_{\varphi}$ assigns to each small edge a density equal to the number of its preimages in $T$. The resulting measure clearly satisfies the balance condition (2.1) at all vertices. Condition (6) implies that we can arrange $\varphi$ so that $m_{\varphi} \in \mathcal{M}_{r}$ provided that $r$ is sufficiently large (so that $\triangle_{r}$ contains $\varphi(V)$ whenever $V$ is a vertex of order 3 of $T$ ). A measure $m \in \mathcal{M}_{r}$ will be called a tree measure if $m=m_{\varphi}$ for some immersion $\varphi$ of a tree. The following illustration shows a tree, and the range of one of its immersions. The arrows indicates ends of the tree, and the asterisk indicates where one of these ends is mapped by the immersion.

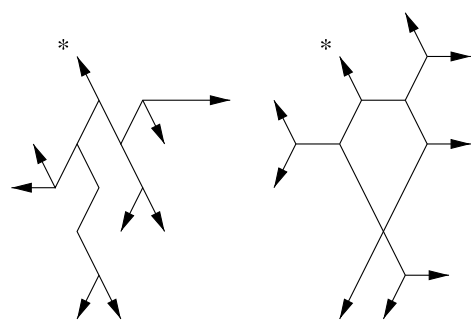

In the second illustration, some edges of the immersion have multiplicity two (i.e., they have two preimages under the corresponding immersion). They are represented by thicker lines.

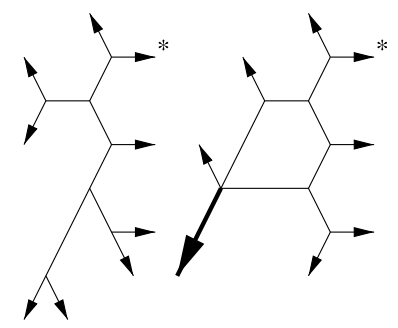


Here is one more figure illustrating the fact that a tree measure need not be extremal.
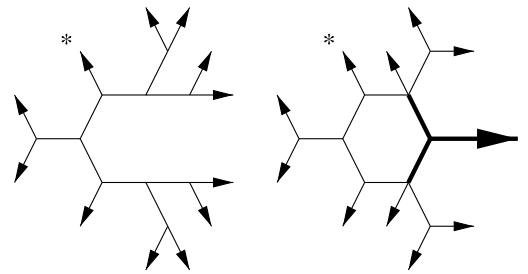

In this case, the measure $m_{\varphi}$ has two summands with unit densities; the support of one of them is pictured below.

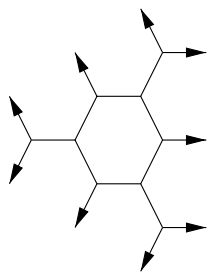

If $m \in \mathcal{M}_{r}$ is a tree measure, it is fairly easy to see that the number of ends of the corresponding tree $T$ is $3 \omega(m)$. We will write $\omega(T)=\omega(m)$. For the trees above, the value of $\omega(T)$ is 3 or 4 .

In the following result we rely on the concepts introduced in [1], particularly the concept of an evil path used in the proof of the second statement. For the convenience of the reader, we recall that a sequence $A_{0} A_{1} \cdots A_{n}$ of lattice vertices is called an evil path (relative to a measure $m$ ) if each segment $A_{j-1} A_{j}$ is a small edge in the support of $m$, and $A_{j-1} A_{j} A_{j+1}$ is an evil turn for $1 \leq j<n$. For the paths considered in the proof, only two kinds of evil turn are needed. The first is no turn at all, that is, $A_{j-1}, A_{j}$ and $A_{j+1}$ are collinear and $A_{j-1} \neq A_{j+1}$. The second is such that $\varangle A_{j-1} A_{j} A_{j+1}=120^{\circ}$ and the small edge $A_{j} X$ such that $\varangle A_{j-1} A_{J} X=\varangle X A_{j} A_{j+1}=120^{\circ}$ is also in the support of $m$.

The second statement of this result settles a basic question about tree measures. The concept of a root edge is explained in the proof.

Theorem 3.1 Assume that $m \in \mathcal{M}_{r}$ is a rigid extremal measure. Then there exists a tree measure $m^{\prime} \in \mathcal{M}_{r}$ such that $m=\mathrm{cm}^{\prime}$ for some constant $c>0$. Conversely, every rigid tree measure is extremal and it assigns unit density to its root edges.

Proof Assume, more generally, that $m \in \mathcal{M}_{r}$ is a rigid measure. Given two adjacent small edges $A B, B C$ in the support of $m$, we write $A B \rightarrow_{m} B C$ if either

(a) $A, B, C$ are collinear and one of the edges $B X$ such that $\varangle X B C=60^{\circ}$ satisfies $m(B X)=0$, or

(b) $\varangle A B C=120^{\circ}$, and the edge $B X$ opposite $A B$ satisfies $m(B X)=0$.

Given an edge $e=A B$, there exist at most two edges $f$ adjacent to $B$ such that $e \rightarrow_{m} f$. More generally, if $e, f$ are two small edges, we write $e \Rightarrow_{m} f$ if either 
$e=f$, or

$$
e=e_{1} \rightarrow_{m} e_{2} \rightarrow_{m} \cdots \rightarrow_{m} e_{k}=f
$$

for some chain $\gamma=\left\{e_{1}, e_{2}, \ldots, e_{k}\right\}, e_{j}=X_{j-1} X_{j}$, of small edges. This relation is called descendance, and it was proved in [1] that each edge in the support of $m$ is the descendant of a minimal (or root) edge contained in $\triangle_{r}$. Moreover, the descendants of a root edge form the support of an extremal measure. Here minimality is defined up to the equivalence relation $e \Leftrightarrow_{m} f$ if $e \Rightarrow_{m} f$ and $f \Rightarrow_{m} e$. A chain $\gamma$ as above is called a descendance path from $e$ to $f$.

Assume now that $m$ is extremal and $e$ is a root edge for $m$ contained in $\triangle_{r}$. Dividing $m$ by $c=m(e)$, we may assume that $m(e)=1$. If $f$ is any edge in the support of $m, m(f)$ equals the number of descendance paths from $e$ to $f$ (cf. [1]). Note that $m$ may have several (often, infinitely many) root edges $f$; they are characterized by the equality $m(f)=1$.

The construction of the required tree $T$ is somewhat analogous to the construction of a universal covering space. Abstractly, the vertices of $T$ are sequences $X_{0} X_{1} \cdots X_{n}$ such that either $n=0$ and $X_{0}$ is an endpoint of $e$, or $n \geq 1$ and $\gamma=\left\{X_{0} X_{1}, X_{1} X_{2}, \ldots, X_{n-1} X_{n}\right\}$ is a descendance path from $e$. The vertices $X_{0}, X_{1}$ are identified with $X_{1} X_{0}, X_{0} X_{1}$, respectively, if $X_{0}$ and $X_{1}$ are the endpoints of $e$. Two vertices of the form $X_{0} X_{1} \cdots X_{n}, X_{0} X_{1} \cdots X_{n} X_{n+1}$ are joined by an edge. There is a map $\varphi: T \rightarrow \mathbb{R}^{2}$ which sends a vertex $X_{0} X_{1} \cdots X_{n}$ to $X_{n}$. We assign now a label $j$ to each edge $e$ of $T$ so that $\varphi(e)$ is parallel to $w_{j}$. It is clear that this labeling satisfies the requirements in the above definition. It should be clear now that $m=m_{\varphi}$, thus proving the first part of the statement.

Consider now an immersion $\varphi$ of a tree $T$ with the property that $m_{\varphi}$ is rigid. Assume $A_{0} A_{1} \cdots A_{n}$ is a simple path in $T$, that is, each $A_{j}$ is a vertex of $T, A_{j-1} A_{j}$ is an edge of $T$ for $j \geq 1$, and $A_{j+1} \neq A_{j-1}$ for $1 \leq j \leq n-1$. The immersion $\varphi$ maps this path onto an evil path in the support of $m_{\varphi}$ [1]. Since $m_{\varphi}$ is rigid, its support contains no evil loops, and therefore we cannot have $\varphi\left(A_{n-1}\right)=\varphi\left(A_{0}\right)$ and $\varphi\left(A_{n}\right)=\varphi\left(A_{1}\right)$. Also observe that every descendance path is the image under $\varphi$ of a simple path in $T$. (This last fact is the appropriate version of the path lifting property of the universal cover.)

Consider next a path $A_{0} A_{1} \cdots A_{n}$ in $T$ with the property that $n \geq 3, \varphi\left(A_{0} A_{1}\right)$ and $\varphi\left(A_{n-1} A_{n}\right)$ are root edges of $m_{\varphi}$, but $\varphi\left(A_{j-1} A_{j}\right)$ is not a root edge for any $j=2,3, \ldots, n-1$. When $n \geq 3$ we must have $\varphi\left(A_{1} A_{2}\right) \nrightarrow_{m_{\varphi}} \varphi\left(A_{0} A_{1}\right)$, and we conclude that the two small edges adjacent to $\varphi\left(A_{1}\right)$, and forming $60^{\circ}$ angles with $\varphi\left(A_{0} A_{1}\right)$, are in the support of $m_{\varphi}$. A similar argument about $\varphi\left(A_{n-1} A_{n}\right)$ shows that

$$
\varphi\left(A_{1}\right) \varphi\left(A_{2}\right) \cdots \varphi\left(A_{n-1}\right) \varphi\left(A_{n}\right) \varphi\left(A_{n-1}\right) \cdots \varphi\left(A_{2}\right) \varphi\left(A_{1}\right)
$$

is an evil loop in the support of $m_{\varphi}$, contrary to the assumption that $m_{\varphi}$ is rigid. When $n=2, \varphi\left(A_{0} A_{1}\right) \not \rightarrow \varphi\left(A_{1} A_{2}\right)$, and $\varphi\left(A_{1} A_{2}\right) \not \rightarrow \varphi\left(A_{0} A_{1}\right)$ we conclude that all the six edges adjacent to $\varphi\left(A_{1}\right)$ are in the support of $m_{\varphi}$, also contrary to rigidity.

The preceding argument proves the following fact. If $A B$ and $C D$ are two edges of $T$ such that $\varphi(A B)$ and $\varphi(C D)$ are root edges of $m_{\varphi}$, then $\varphi(X Y)$ is also a root edges of $m_{\varphi}$ for every $X Y$ on the shortest path joining $A B$ and $C D$. Moreover, all 
these root edges are equivalent relative to descendance. Now, every root edge of $m_{\varphi}$ is the image of some edge in $T$, and any two edges in $T$ are joined by a simple path. We conclude that all the root edges of $m_{\varphi}$ are equivalent, and therefore $m_{\varphi}$ is extremal.

It remains to show that $m_{\varphi}$ assigns unit mass to its root edges. Assume to the contrary that there exist two distinct edges in $T$ which are mapped to the same root edge of $m_{\varphi}$. Thus we can find a simple path $A_{0} A_{1} \cdots A_{n}$ in $T$ such that each edge $A_{j-1} A_{j}$ is mapped to a root edge, and $\varphi\left(A_{0} A_{1}\right)=\varphi\left(A_{n-1} A_{n}\right)$. We choose such a path with minimum $n$, so that all the other edges $\varphi\left(A_{j-1} A_{j}\right)$ are distinct. The preceding observations about evil loops imply that $\varphi\left(A_{0}\right)=\varphi\left(A_{n}\right)$ and $\varphi\left(A_{1}\right)=\varphi\left(A_{n-1}\right)$. The edges $\varphi\left(A_{1} A_{2}\right)$ and $\varphi\left(A_{n-2} A_{n-1}\right)$ meet at $\varphi\left(A_{n-1}\right)$, and they cannot form a $60^{\circ}$ degree angle because $\varphi\left(A_{n-1} A_{n}\right)$ is a root edge. It follows that they form a $120^{\circ}$ angle, and therefore the loop $\varphi\left(A_{1}\right) \varphi\left(A_{2}\right) \cdots \varphi\left(A_{n-1}\right)$ is evil, a contradiction.

Let $\varphi$ be the immersion of $T$ described in the preceding proof, and let $e$ be an edge of $T$ such that $\varphi(e)$ is a root edge for the measure $m$. We can orient all other edges of $T$ away from $e$. It was shown in [1] that the map $\varphi$ has the following additional property: if $g$ and $h$ are two edges such that $\varphi(g)=\varphi(h)$, then $\varphi$ induces the same orientation on this common image. In other words, the edges in the support of $m$, other than $\varphi(e)$, can be consistently oriented in the direction of a descendance path from $\varphi(e)$. The following lemma is also proved in [1] (see the discussion following Theorem 3.5 in [1]).

Lemma 3.2 Let $m$ be a rigid extremal measure, and orient the edges in its support away from a fixed root edge. Each lattice point meets at most four edges in the support of $m$, and the possible positions of these edges, including their orientations, are as follows

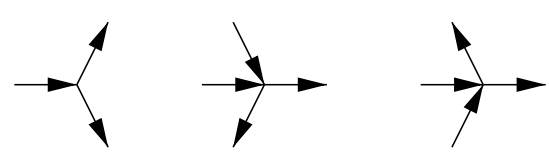

up to rotations.

In order to study the sums $\Sigma_{m^{\prime}}(m)$, we will also need some maps which are closely related to immersions, but are discontinuous. Assume that $T$ is a tree, and $\varphi$ is an immersion of $T$ such that the induced measure is in $\mathcal{M}_{r}$ for some $r$. Denote by $T_{\circ}$ the set of points in $T$ which are not vertices. A function $\psi: T_{\circ} \rightarrow \mathbb{R}^{2}$ will be called a fractured immersion if

(1) the range of $\psi$ is contained in the small edges of the triangular lattice determined by $w_{1}, w_{2}, w_{3}$,

(2) there is an immersion $\varphi$ of $T$ such that $\psi(t)-\varphi(t)$ is constant on the interior of every edge, and

(3) $\psi$ extends continuously to all except finitely many vertices of $T$.

Let $\psi$ be a fractured immersion of a tree $T$. We will associate to each vertex $V$ of $T$ an integer $\delta_{\psi}(V)$ which measures how badly fractured $\psi$ is at $V$. If $\psi$ extends continuously to the point $V$ we set $\delta_{\psi}(V)=0$. Assume next that the order of $V$ is 2 
and the two edges $A V, V B$ are mapped to $A^{\prime} V^{\prime}, V^{\prime \prime} B^{\prime}$, respectively, with $V^{\prime} \neq V^{\prime \prime}$. We will set $\delta_{\psi}(V)=q$ if the point $V^{\prime \prime}$ lies $q$ lattice units to the left of the line joining $A^{\prime}$ and $V^{\prime}$, where this line is oriented so that $A^{\prime} V^{\prime}$ points toward $V^{\prime}$. Note that $V^{\prime \prime}$ could be to the right of this line, in which case $q<0$, and $V^{\prime \prime}$ (as well as $B^{\prime}$ ) could be on this line, in which case $q=0$. Finally, let $V$ be a vertex of order 3, assume that the three edges $A V, B V, C V$ are mapped to $A^{\prime} V^{\prime}, B^{\prime} V^{\prime \prime}, C^{\prime} V^{\prime \prime \prime}$, and note that these three segments still form $120^{\circ}$ angles. If the lines containing these three segments are concurrent, we set $\delta_{\psi}(V)=0$. Otherwise, these three lines form an equilateral triangle $\triangle$ with side length $q$. Orient the sides of this triangle so that the segments $A^{\prime} V^{\prime}, B^{\prime} V^{\prime \prime}, C^{\prime} V^{\prime \prime \prime}$ point toward $V^{\prime}, V^{\prime \prime}, V^{\prime \prime \prime}$, respectively. If the boundary of $\triangle$ is oriented clockwise, set $\delta_{\psi}(V)=-q$, and in the contrary case set $\delta_{\psi}(V)=q$. The following figures shows three cases in which the values of $\delta_{\psi}(V)$ are $0,-2$ and 1 . The dotted lines represent small edges.
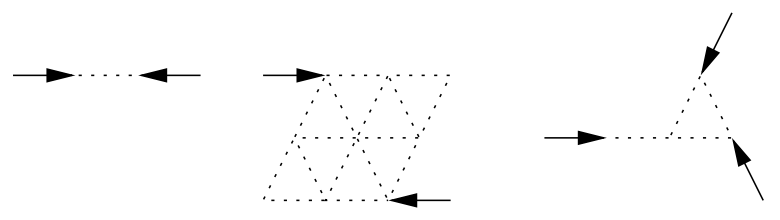

The orientations indicated above are used exclusively for the calculation of the numbers $\delta_{\psi}(V)$. In the proofs below we will need to orient all the edges of a tree $T$ (not just the ones adjacent to $V$ ), and this will generally be the orientation away from a fixed vertex or edge.

In the following statement, the segment $A_{0} X_{0}$ is deemed to exit $\triangle_{r}$ at the point $A_{0}$, rather than $C_{r}$, while $C_{r} Z_{r}$ is deemed to exit at $C_{r}$. Of course, this issue does not arise when the corners of $\triangle_{r}$ are not exit points, and this can be achieved by enlarging the triangle.

Theorem 3.3 Let $\psi$ be a fractured immersion of a tree $T$ such that all the limits of $\psi$ at discontinuity points are contained in $\triangle_{r}$. For each end $E$ of $T$, denote by $\ell(E)$ the rank of the exit point of $\psi(E)$ from $\triangle_{r}$. In other words, $\ell(E)=\ell$ if the closure of $\psi(E)$ intersects $\partial \triangle_{r}$ in $A_{\ell}, B_{\ell}$, or $C_{\ell}$. Then we have

$$
\sum_{\text {all ends } E \text { of } T} \ell(E)=r \omega(T)+\sum_{\text {all vertices } V \text { of } T} \delta_{\psi}(V) .
$$

Proof We proceed by induction on the number of vertices where $\psi$ does not extend continuously. When this number is equal to zero, $g$ is an immersion, and the sum in the left hand side is nothing but

$$
\sum_{\ell} \ell\left(\alpha_{\ell}+\beta_{\ell}+\gamma_{\ell}\right)=r \omega(m)
$$

where $\alpha_{\ell}, \beta_{\ell}, \gamma_{\ell}$ are the exit densities of the corresponding measure $m$. This is precisely the desired identity because $\omega(m)=\omega(T)$. Assume then that the theorem has been proved for all fractured immersions with fewer discontinuity points than $\psi$, and 
there exists at least one vertex $V$ where $\psi$ does not extend continuously. Consider first the case when $V$ is of order 2, and the vertices $A V, V B$ are mapped by $\psi$ to $A^{\prime} V^{\prime}, V^{\prime \prime} B^{\prime}$, which we will assume to be horizontal for definiteness. By transposing the points $A, B$, we can also assume that $A^{\prime}$ is to the left of $V^{\prime}$ and $B^{\prime}$ is to the right of $V^{\prime \prime}$. There is then a point $A_{\ell}$ such that the segment $A_{\ell} V^{\prime \prime}$ is horizontal; denote by $a$ its length. Similarly, there is a point $C_{k}$ such that the segment $V^{\prime} C_{k}$ is horizontal. The definition of $\delta$ implies that

$$
\ell+k+\delta_{\psi}(V)=r
$$

We now form two trees in the following way. Cut the tree $T$ at the point $V$, and add to the part containing $A V$ an end $V V_{1} V_{2} \cdots$, thus forming a tree $T^{\prime}$. Analogously, add to the part containing $B V$ a path $V W_{1} W_{2} \cdots W_{a}$, where $W_{1}, W_{2}, \ldots, W_{a-1}$ have order 2, and two ends meeting at $W_{a}$, thus forming a tree $T^{\prime \prime}$. The map $\psi$ gives rise to two fractured immersions $\psi^{\prime}$ and $\psi^{\prime \prime}$ of $T^{\prime}$ and $T^{\prime \prime}$ as follows: $\psi^{\prime}\left(V V_{1} V_{2} \cdots\right)$ is the half line starting with $A^{\prime} V^{\prime}, \psi^{\prime \prime}\left(V W_{1} W_{2} \cdots W_{a}\right)=V^{\prime \prime} A_{\ell}$, and the two ends meeting at $W_{a}$ are mapped onto the two half lines starting at $A_{\ell}$ and pointing left. It is clear that $\psi^{\prime}$ and $\psi^{\prime \prime}$ have fewer vertices of discontinuity than $\psi$, and therefore the desired formula is true for $\psi^{\prime}$ and $\psi^{\prime \prime}$. It is clear that

$$
\sum_{\text {vertices } W \text { of } T} \delta_{\psi}(W)=\sum_{\text {vertices } V^{\prime} \text { of } T^{\prime}} \delta_{\psi^{\prime}}\left(V^{\prime}\right)+\sum_{\text {vertices } V^{\prime \prime} \text { of } T^{\prime \prime}} \delta_{\psi^{\prime \prime}}\left(V^{\prime \prime}\right)+\delta_{\psi}(V),
$$

while

$$
\sum_{\text {all ends } E^{\prime} \text { of } T^{\prime}} \ell\left(E^{\prime}\right)+\sum_{\text {all ends } E^{\prime \prime} \text { of } T^{\prime \prime}} \ell\left(E^{\prime \prime}\right)=\sum_{\text {all ends } E \text { of } T} \ell(E)+\ell+k .
$$

The desired equality follows then from (3.1) because $\omega\left(T^{\prime}\right)+\omega\left(T^{\prime \prime}\right)=\omega(T)+1$. The solid arrows in the following illustration are the oriented segments $A^{\prime} V^{\prime}$ and $B^{\prime} V^{\prime \prime}$, while the dashed lines indicate where the additional edges in $T^{\prime}$ and $T^{\prime \prime}$ are mapped. Their exit points from $\triangle_{r}$ are $A_{\ell}, B_{0}$ and $C_{k}$.

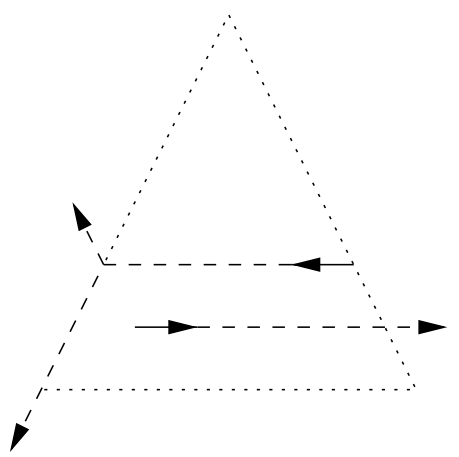

Consider next the case that $V$ is of order three, and the three edges $A V, B V, C V$ of $T$ are mapped to $A^{\prime} V^{\prime}, B^{\prime} V^{\prime \prime}, C^{\prime} V^{\prime \prime \prime}$. Assume that $A, B, C$ are arranged clockwise around $V$. A cyclic permutation allows us to assume that $A^{\prime} V^{\prime}$ is horizontal, and we 
must consider the two cases where $A^{\prime}$ is to the left or to the right of $V^{\prime}$. These two situations are illustrated below.
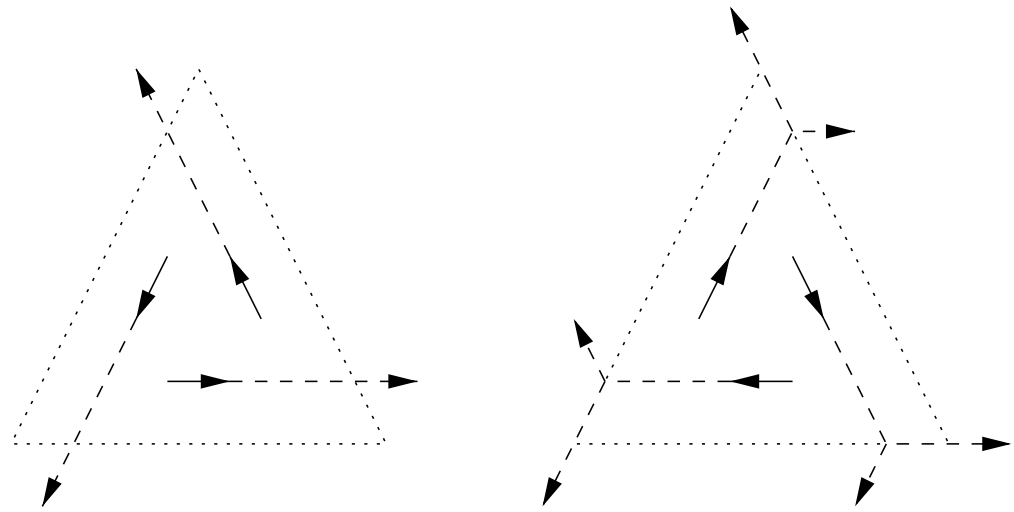

Assume first that $A^{\prime}$ is on the left. The half lines $A^{\prime} V^{\prime}, B^{\prime} V^{\prime \prime}, C^{\prime} V^{\prime \prime \prime}$ exit $\triangle_{r}$ at points $C_{k}, B_{\ell}, A_{p}$, respectively. As in the preceding proof, we cut $T$ at the point $V$, and form three trees $T^{\prime}, T^{\prime \prime}, T^{\prime \prime \prime}$ by attaching to the part of $T$ which contains $A, B, C$, respectively, an end attached at $V$. The map $\psi$ gives rise to three fractured immersions $\psi^{\prime}, \psi^{\prime \prime}, \psi^{\prime \prime \prime}$ of these trees. For instance, $\psi^{\prime}$ maps the additional end at $V$ to the half line starting with $V^{\prime} C_{k}$. Moreover, the new fractured immersions have fewer discontinuity points than $\psi$, and therefore the inductive hypothesis applies to them. As in the preceding case, we have $\omega\left(T^{\prime}\right)+\omega\left(T^{\prime \prime}\right)+\omega\left(T^{\prime \prime \prime}\right)=\omega(T)+1$, and

$$
k+\ell+p+\delta_{\psi}(V)=r .
$$

The desired formula follows now easily. Finally, consider the case in which $A^{\prime}$ is to the right of $V^{\prime}$. In this case, the half lines $A^{\prime} V^{\prime}, B^{\prime} V^{\prime \prime}, C^{\prime} V^{\prime \prime \prime}$ exit $\triangle_{r}$ at points $A_{\ell}, B_{k}, C_{p}$, respectively, and the trees $T^{\prime}, T^{\prime \prime}, T^{\prime \prime \prime}$ must be constructed by attaching at $V$ a few edges followed by two ends. In this case we have $\omega\left(T^{\prime}\right)+\omega\left(T^{\prime \prime}\right)+\omega\left(T^{\prime \prime \prime}\right)=$ $\omega(T)+2$ and the reader can verify easily that $k+\ell+p+\delta_{\psi}(V)=2 r$. The conclusion follows as before.

\section{Inflations and fractured immersions}

We recall from [8] (see also [1]) that every measure $v \in \mathcal{M}_{r}$ has an associated puzzle obtained by inflating $v$. The inflation of $v$ is defined as follows. Cut the plane along the edges in the support of $v$ to obtain a collection of puzzle pieces, and translate these pieces away from each other in the following way: the parallelogram formed by the two translates of a side $A B$ of a white puzzle piece has two sides of length equal to the density of $v$ on $A B$ and $60^{\circ}$ clockwise from $A B$. This parallelogram will be referred to as the inflation of $A B$. The balance condition (2.1) implies that the original puzzle pieces and these parallelograms fit together, and leave a space corresponding to each branch point in the support of $v$. Here is an illustration of the process with $r=3$; the thinner lines in the support of the measure have density one, and the thicker ones 
density 2. The original pieces of the triangle $\Delta_{r}$ are white, the added parallelogram pieces are dark gray, and the branch points become light gray pieces. Each light gray piece has as many sides as there are branches at the original branch point.
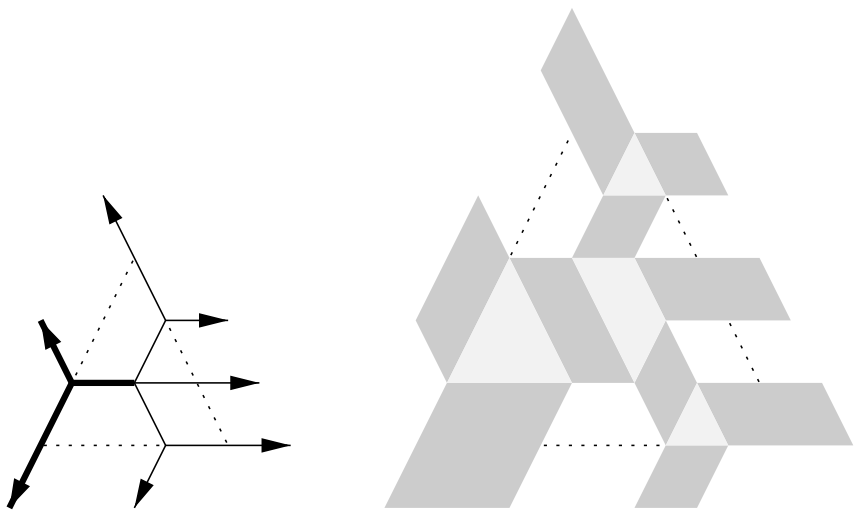

The dotted lines indicating the boundary of $\Delta_{r}$ have been translated so that they now outline a triangle with sides $r+\omega(v)$, which we may assume is precisely $\triangle_{r+\omega(v)}$. The decomposition of this triangle into white, dark gray, and light gray pieces is the puzzle associated to $v$. The white regions in the puzzle are called 'zero regions', the light gray ones 'one regions', and the dark gray parallelograms ' $0-1$ regions' in [8]. Observe that knowledge of the dark gray parallelograms in a puzzle determines the colors of the remaining pieces. Indeed, the white sides of such a parallelogram, that is, the sides bordering white puzzle pieces, are $60^{\circ}$ counterclockwise from its light gray sides. The figure symmetric to a puzzle relative to a vertical line is also a puzzle provided that the white and light gray colors are interchanged.

The puzzle of a measure $v \in \mathcal{M}_{r}$ can be used to define a dual measure $v^{*} \in \mathcal{M}_{\omega(v)}^{*}$. This measure is obtained as follows. Replace each dark gray parallelogram in the puzzle of $v$ by a line segment parallel to its light gray sides, and assign this segment density equal to the white side of the same parallelogram. Now move the light gray pieces together in such a way that they cover $\triangle_{\omega(v)}$ and the new segments form the boundary of the light gray pieces. Then these segments and their densities define the part of the measure $v^{*}$ supported in $\triangle_{\omega(v)}$; the part of the measure outside this triangle, that is, the exit densities, is determined by the balance condition. The process of passing from a puzzle to the dual measure is called $*$-deflation. It is easy to see that the exit densities of $v^{*}$ are entirely determined by the exit densities of $v$. Moreover the processes of inflation and $*$-deflation can be reversed, leading to the conclusion that $c_{\nu^{*}}=c_{\nu}$. This duality plays an essential role in Sect. 7. The following figure illustrates the dual of the measure pictured above.

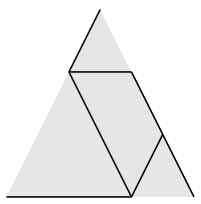


It is useful to observe that the measure $\mu^{*}$ can be obtained directly from $\mu$, without passing through the puzzle. Start with a segment $A B \subset \triangle_{r}$ in the support of $\mu$ with no branch points of $\mu$ in its interior. Assume that $A B$ has length $\ell$ and $\mu$ has density $\delta$ on $A B$. Replace $A B$ with a segment $A^{\prime} B^{\prime}$ which is $60^{\circ}$ clockwise from $A B$, the length of $A^{\prime} B^{\prime}$ is $\delta$, and assign it density $\ell$. Arrange then these segments in such a way that the sides $A B, B C, \ldots$ of a white puzzle piece correspond to edges with a common endpoint. Then the union of these new segments is the support of $\mu^{*}$ in $\triangle_{\omega(\mu)}$ (or, more precisely, a translate of this support), and the new densities are the ones assigned by $\mu^{*}$. When viewed as a planar graph, the support of $\mu^{*}$ is dual to that of $\mu$. More precisely, the support of $\mu^{*}$ in $\triangle_{\omega(\mu)}$ can be obtained as follows, up to an orientation preserving homeomorphism. Place one point in each connected component of $\triangle_{r} \backslash \operatorname{supp}(\mu)$, and in addition, for every side $A B$ of such a component in $\partial \triangle_{r} \cap \operatorname{supp}(\mu)$, place a point on the outside of $\triangle_{r}$, close to $A B$. Now join two such points if they are in components of $\mathbb{R}^{2} \backslash \operatorname{supp}(\mu)$ sharing a side. The length of this segment $X Y$ should equal the density of the edge $A B$ separating the two corresponding regions (where the points $X$ and $Y$ were placed), while its density in $m^{*}$ is equal to the length of $A B$. The following figure helps illustrate this duality.
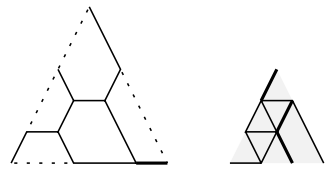

We recall one further concept introduced in [1]. Two measures $m$ and $\tilde{m}$ are said to be homologous if there is a bijection between the white piece edges determined by the support of $m$ and those determined by the support of $\tilde{m}$ such that corresponding edges are parallel, and incident edges correspond to incident edges (the intersection point being the one dictated by the correspondence of the edges). The preceding discussion makes it clear that the duals of homologous measures are also homologous. Moreover, if $m$ and $\tilde{m}$ assign the same density to homologous edges, then $\omega(m)=\omega(\tilde{m})$ and the measures $m^{*}$ and $\tilde{m}^{*}$ have precisely the same support.

In this section, the main use of inflations is to produce fractured immersions from a given immersion of a tree $T$. Assume indeed that $T$ is a tree, $\varphi$ is an immersion of $T$ such that the corresponding measure $m^{\prime}=m_{\varphi}$ is in $\mathcal{M}_{r}$, and let $v \in \mathcal{M}_{r}$ be another measure. Assume that each edge of $T$ has been given an orientation, and that all the edges belonging to an end of $T$ have been oriented outward (i.e., toward the infinite part of that end). For each edge $e$ in $T$ such that $\varphi(e)$ is in the support of $v$, we attach $\varphi(e)$ to the white puzzle piece on the right of $\varphi(e)$ when $\varphi(e)$ is given the orientation induced by the orientation of $e$. For edges $e$ with $v(e)=0$, $\varphi(e)$ is contained in a white puzzle piece, and it moves along with that piece. If we denote now by $\psi(e)$ the translate of $\varphi(e)$ in the puzzle construction, we obviously obtain a fractured immersion. The following figure illustrates the process as applied to a measure $m^{\prime}=m_{\varphi}$ whose support is pictured below, and $v$ is the measure whose inflation was depicted in the preceding figure. We have oriented all the edges away 
from the branch point inside $\triangle_{3}$, and completed the outline of $\triangle_{6}$.
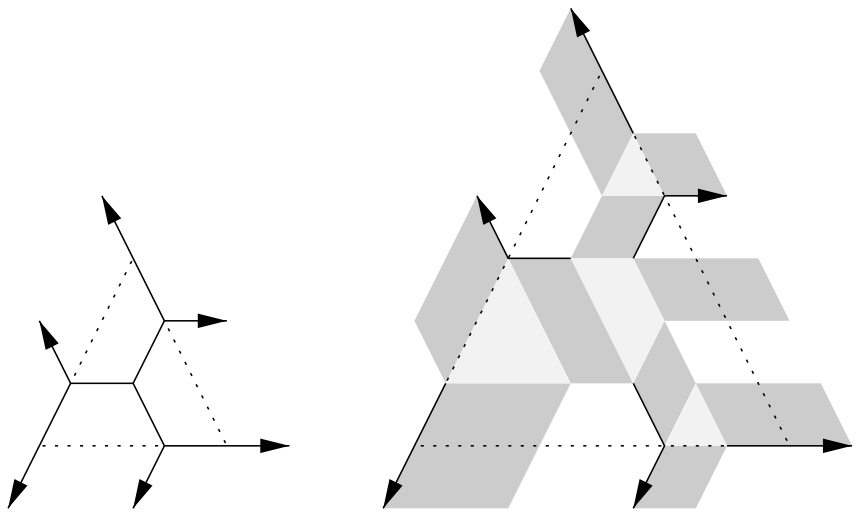

Note that all the fractures of $\psi$ are contained in $\triangle_{r+\omega(v)}$, and therefore the formula in Theorem 3.3 applies. Let $\alpha_{j}, \beta_{j}, \gamma_{j}$ be the exit densities of $\nu$, and let $\alpha_{j}^{\prime}, \beta_{j}^{\prime}, \gamma_{j}^{\prime}$ be the exit densities of $m^{\prime}$. Then it is easy to see that

$$
\begin{aligned}
\sum_{\text {all ends } E \text { of } T} \ell(E) & =\sum_{\ell=0}^{r}\left[\alpha_{\ell}^{\prime}\left(\ell+\sum_{k<\ell} \alpha_{k}\right)+\beta_{\ell}^{\prime}\left(\ell+\sum_{k<\ell} \beta_{k}\right)+\gamma_{\ell}^{\prime}\left(\ell+\sum_{k<\ell} \gamma_{k}\right)\right] \\
& =r \omega\left(m^{\prime}\right)+\sum_{k<\ell}\left(\alpha_{\ell}^{\prime} \alpha_{k}+\beta_{\ell}^{\prime} \beta_{k}+\gamma_{\ell}^{\prime} \gamma_{k}\right) .
\end{aligned}
$$

Indeed, this follows from the fact that an end $E$ such that $\varphi(E)$ exits at $A_{\ell}$ is translated to $\psi(E)$ which exits at $A_{\ell+\alpha_{0}+\cdots+\alpha_{\ell-1}}$.

Lemma 4.1 With the notation above, we have

$$
\Sigma_{m^{\prime}}(v)=\sum_{\text {all vertices } V \text { of } T} \delta_{\psi}(V) .
$$

Proof Theorem 3.3 yields

$$
\sum_{\text {all ends } E \text { of } T} \ell(E)=(r+\omega(v)) \omega\left(m^{\prime}\right)+\sum_{\text {all vertices } V \text { of } T} \delta_{\psi}(V) .
$$

Combining this with the identity preceding the statement, we obtain

$$
\sum_{\text {all vertices } V \text { of } T} \delta_{\psi}(V)=\sum_{k<\ell}\left(\alpha_{\ell}^{\prime} \alpha_{k}+\beta_{\ell}^{\prime} \beta_{k}+\gamma_{\ell}^{\prime} \gamma_{k}\right)-\omega\left(m^{\prime}\right) \omega(v),
$$

and this is precisely the formula (2.3) for $\Sigma_{m^{\prime}}(v)$.

Theorem 4.2 Assume that $v, m^{\prime} \in \mathcal{M}_{r}$, and $m^{\prime}$ is a tree measure.

(1) If the support of $m^{\prime}$ is not contained in the support of $v$, then $\Sigma_{m^{\prime}}(v) \geq 0$. 
(2) If $m^{\prime}$ is not rigid, we also have $\Sigma_{m^{\prime}}\left(m^{\prime}\right) \geq 0$.

(3) If $m^{\prime}$ is rigid, we have $\Sigma_{m^{\prime}}\left(m^{\prime}\right)=-1$.

Proof Let $\varphi$ be an immersion of a tree $T$ such that $m^{\prime}=m_{\varphi}$. To prove (1), fix an edge $e_{0}$ such that $\varphi\left(e_{0}\right)$ is not contained in the support of $\nu$, and orient all the other edges of $T$ away from $e_{0}$. Construct a fractured immersion $\psi$ using the above construction associated with the inflation of $v$. It is easy to verify that in this case we have $\delta_{\psi}(V) \geq 0$ for every vertex $V$ of $T$. Indeed, $\delta_{\psi}(V)$ can be calculated explicitly in terms of the values of $v$ on one of the edges adjacent to $\varphi(V)$. To see this, assume first that $V$ is of order two, $A V$ and $V B$ are the two adjacent edges, and they are mapped by $\varphi$ to $A^{\prime} V^{\prime}$ and $V^{\prime} B^{\prime}$. These two edges are shown below, with the arrows indicating their orientation, and the dotted extensions are drawn to indicate the value of $\delta_{\psi}(V)$.

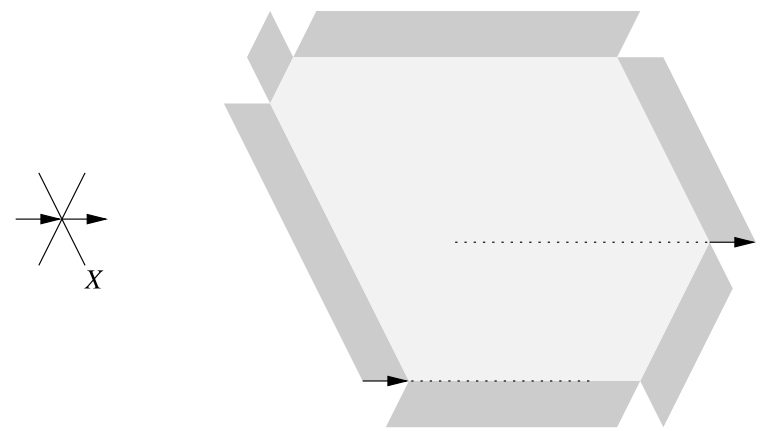

Clearly, we have $\delta_{\psi}(V)=v\left(V^{\prime} X\right)$, with $X$ as in the figure, i.e. on the right side of $A^{\prime} V^{\prime}$, and $\varangle X V^{\prime} B^{\prime}=60^{\circ}$. If $V$ has order three, let $A V, B V, C V$ be the three adjacent edges, with $A V$ oriented toward $V$. Assume that $\varphi(A V)=A^{\prime} V^{\prime}$, and $X$ is symmetric to $A^{\prime}$ relative to $V^{\prime}$. We have again $\delta_{\psi}(V)=v\left(V^{\prime} X\right)$.

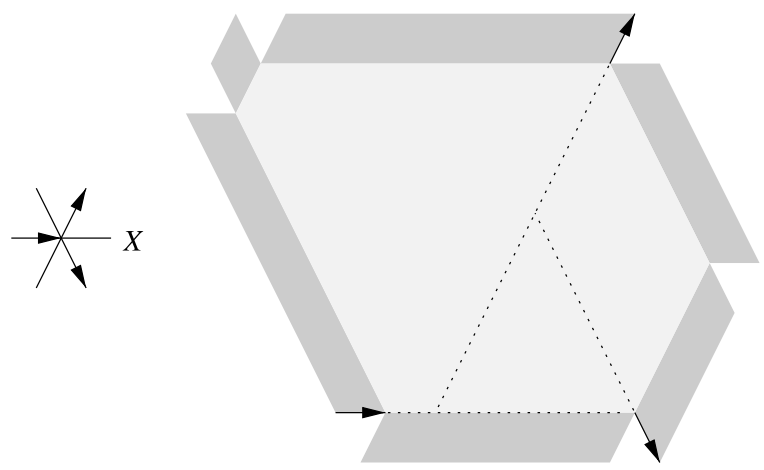

Assertion (1) follows now from Lemma 4.1. (In both illustrations we assumed that $v$ assigns nonzero densities to all six edges adjacent to $V^{\prime}$. More precisely, these densities were taken to be 1,7, 4,3,5 and 6 in clockwise order.)

Assume next that $m^{\prime}$ is not rigid, and choose a different measure $v$ with the same exit densities. Then $v$ can be written as a sum of distinct extreme measures, say 
$v=\sum_{j} m_{j}$. If the support of $m_{j}$ contains the support of $m^{\prime}$, then $m_{j}$ is a positive multiple of $m^{\prime}$ by extremality. Thus there is at most one $j$ such that the support of $m_{j}$ contains the support of $m^{\prime}$. Assume for definiteness that $m_{1}=\kappa m^{\prime}$, where $0 \leq \kappa<1$. Then part (1) of the theorem implies that $\Sigma_{m^{\prime}}\left(m_{j}\right) \geq 0$ for $j \neq 1$, hence

$$
\Sigma_{m^{\prime}}\left(m^{\prime}\right)=\Sigma_{m^{\prime}}(v)=\sum_{j} \Sigma_{m^{\prime}}\left(m_{j}\right) \geq \Sigma_{m^{\prime}}\left(m_{1}\right)=\kappa \Sigma_{m^{\prime}}\left(m^{\prime}\right),
$$

and therefore $\Sigma_{m^{\prime}}\left(m^{\prime}\right) \geq 0$, as claimed.

Finally, assume that $m^{\prime}$ is rigid, and recall from Theorem 3.1 that $m^{\prime}$ assigns unit mass to its root edges. Choose an edge $e_{0}$ such that $\varphi\left(e_{0}\right)$ is a root edge for $v=m^{\prime}$ contained in $\triangle_{r}$. Orient the other edges $T$ away from $e_{0}$, and also give $e_{0}$ some orientation, say it is oriented away from one of its endpoints $V_{0}$. In this case we have $\delta_{\psi}\left(V_{0}\right)=-1$ and $\delta_{\psi}(V)=0$ for all other vertices. To verify this fact one must observe that in the pictures above we must have $m^{\prime}\left(V^{\prime} X\right)=0$ because of the rigidity of $m^{\prime}$. This follows from Lemma 3.2. The only exception is the orientation at the point $V_{0}$ which produces a nonzero $\delta_{\psi}\left(V_{0}\right)$. To calculate the value of $\delta_{\psi}\left(V_{0}\right)$, we will further assume that $V_{0}$ is a vertex of order 2 and both edges $A_{0} V_{0}, V_{0} B_{0}$ adjacent to $V_{0}$ are mapped by $\varphi$ to root edges of $m^{\prime}$. This can be achieved by applying a homothety, as seen in the Introduction. Assuming, for instance, that $\varphi\left(A_{0}\right)=A, \varphi\left(B_{0}\right)=B$ and $\varphi\left(V_{0}\right)=V$, we have $m^{\prime}(A V)=m^{\prime}(V B)=1$. If we orient $A_{0} V_{0}$ and $B_{0} V_{0}$ away from $V_{0}$, the inflation process looks as follows:

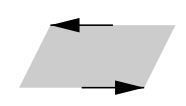

The width of the dark gray parallelogram is $m^{\prime}(A V)=1$, hence $\delta_{\psi}\left(V_{0}\right)=-1$. The theorem follows.

Theorem 3.1 shows that the assumption of part (3) of the preceding result is equivalent to the requirement that $m^{\prime}$ be rigid, extremal, and assign unit density to its root edges. The following result also uses this equivalence.

Corollary 4.3 Assume that $m, m^{\prime} \in \mathcal{M}_{r}$ and $m^{\prime}$ is a rigid tree measure. If $\Sigma_{m^{\prime}}(m)=$ $-p<0$ then $\mathrm{pm}^{\prime} \leq m$ and $c_{m-p m^{\prime}}=c_{m}$.

Proof Let $s$ be the largest number such that $s m^{\prime} \leq m$. Then the support of $m-s m^{\prime}$ does not contain the support of $m^{\prime}$, and therefore $\Sigma_{m^{\prime}}\left(m-s m^{\prime}\right) \geq 0$ by Theorem 4.2(1). Thus

$$
-p=\Sigma_{m^{\prime}}(m)=\Sigma_{m^{\prime}}\left(m-s m^{\prime}\right)+s \Sigma_{m^{\prime}}\left(m^{\prime}\right) \geq-s,
$$

so that $s \geq p$. If $m^{\prime \prime}$ is any other measure with the same exit densities as $m$, it follows that $p m^{\prime} \leq m^{\prime \prime}$ as well, and the exit densities for $m-p m^{\prime}$ and $m^{\prime \prime}-p m^{\prime}$ are the same. This yields a bijection $m^{\prime \prime} \leftrightarrow m^{\prime \prime}-p m^{\prime}$ between measures with the exit densities of $m$ and measures with the exit densities of $m-p m^{\prime}$. 
A different proof of this corollary is given at the end of Sect. 7.

We show later in detail, following the proof of Theorem 6.2, that Corollary 4.3 extends [3, Proposition 3.10]. In our terminology, that results states that $c_{m-m^{\prime}}=c_{m}$ if $m^{\prime}$ is a tree measure with $\omega\left(m^{\prime}\right)=1$, and $\Sigma_{m^{\prime}}(m)<0$. The original formulation in [3] is in terms of the sets $I, J, K$, and the proof proceeds through a very explicit construction of Littlewood-Richardson tableaux.

We can now give a general method for the construction of rigid measures, thus providing the converse of [1, Theorem 3.8]. First, we need to review that result. Let $m \in \mathcal{M}_{r}$ be a rigid measure, and let $m_{1}, m_{2} \in \mathcal{M}_{r}$ be two tree measures with support contained in the support of $m$. The relation $m_{1} \prec_{0} m_{2}$ was defined in [1] as follows: there exist four small edges $A X, X B, C X$ and $X D$ such that

(1) $A X$ and $X B$ are collinear edges in the support of $m_{1}$,

(2) $C X$ and $X D$ are collinear edges in the support of $m_{2}$, and

(3) $X B$ is $60^{\circ}$ clockwise from $X D$.

It was shown in [1] that ' $\prec_{0}$ ' can be extended to an order relation on the set of extremal rigid measures with support contained in the support of $m$. As noted earlier, each extremal rigid measure is a positive multiple of a tree measure. The following result allows us to extend ' $\prec_{0}$ ' to the collection of all rigid tree measures; this extension is no longer contained in an order relation.

Lemma 4.4 Let $m$ be a rigid measure, and let $m_{1}, m_{2}$ be extremal measures with support contained in the support of $m$. We have $m_{1} \prec_{0} m_{2}$ if and only if $\Sigma_{m_{2}}\left(m_{1}\right)>0$.

Proof Observe that $m_{1}$ and $m_{2}$ are also rigid. Let $\varphi$ be an immersion of some tree $T$ such that $m_{2}=m_{\varphi}$; such an immersion exists by Theorem 3.1. Orient all the edges of $T$ away from some edge $e_{0}$ such that $\varphi\left(e_{0}\right)$ is a root edge for $m_{2}$ not contained in the support of $m_{1}$. Assume first that $m_{1} \prec_{0} m_{2}$, and the small edges $A X, X B, C X, X D$ satisfy conditions (1)-(3) above. We may assume that $C X=\varphi\left(e_{1}\right), X D=\varphi\left(e_{2}\right)$, where $e_{1}$ and $e_{2}$ are adjacent edges, and $e_{1}$ is oriented toward $e_{2}$. The proof of Theorem 4.2 implies that $\Sigma_{m_{2}}\left(m_{1}\right) \geq m_{1}(X B)>0$.

Conversely, assume that $\Sigma_{m_{2}}\left(m_{1}\right)>0$. Let $e_{1}, e_{2}, e_{3}$ be three edges of $T$ adjacent to a vertex $V$, and assume that $e_{1}$ is oriented toward $V$. These edges are mapped by $\varphi$ to $A_{j} X, j=1,2,3$, and we must have $A_{1} X \rightarrow_{m} X A_{2}$ and $A_{1} X \rightarrow_{m} X A_{3}$. It follows that the edge $X B$ opposite $A_{1} X$ satisfies $m(X B)=0$, and therefore $m_{1}(X B)=0$, so that this vertex $V$ contributes nothing to $\Sigma_{m_{2}}\left(m_{1}\right)$. We conclude that there must exist some vertex $V$ of order 2 which contributes to $\Sigma_{m_{2}}\left(m_{1}\right)$. Let $e_{1}, e_{2}$ be the two edges adjacent to $V$, and assume that $e_{1}$ is oriented toward $V$. Then $\varphi$ maps these two edges to collinear edges $C X, X D$ so that $C X \rightarrow_{m} X D$. The fact that $V$ contributes to $\Sigma_{m_{2}}\left(m_{1}\right)$ means simply that the edge $X B$ which is $60^{\circ}$ clockwise from $X D$ is in the support of $m_{1}$. We claim that the edge $A X$ opposite $X B$ is also in the support of $m_{1}$. Indeed, the fact that $C X \rightarrow_{m} X D$ implies that the edge $X B^{\prime}$ which is $60^{\circ}$ counterclockwise from $X D$ is not in the support of $m$, hence not in the support of $m_{1}$. The balance condition for $m_{1}$ implies that $m_{1}(A X)>0$. Thus the vertices $A X, X B, C X, X D$ witness the fact that $m_{1} \prec_{0} m_{2}$. 
Corollary 3.6 of [1] allows us to write any rigid measure $m \in \mathcal{M}_{r}$ under the form

$$
m=\sum_{j=1}^{n} p_{j} m_{j},
$$

where $p_{j}>0$, and the $m_{j}$ are distinct rigid extremal tree measures. Moreover, Theorem 3.8 of that paper allows us to arrange the terms of this sum in such a way that $m_{i} \prec_{0} m_{j}$ implies that $i \leq j$. According to Lemma 4.4, $m_{i} \prec_{0} m_{j}$ is equivalent to $\Sigma_{m_{j}}\left(m_{i}\right)>0$ for these measures.

Corollary 4.5 Let $m \in \mathcal{M}_{r}$ be a rigid measure. There exist extremal, rigid tree measures $m_{1}, m_{2}, \ldots, m_{n}$ and positive numbers $p_{1}, p_{2}, \ldots, p_{n}$ such that $m=$ $\sum_{j=1}^{n} p_{j} m_{j}$, and $\Sigma_{m_{i}}\left(m_{j}\right)=0$ for $i<j$. Conversely, assume that $m_{1}, m_{2}, \ldots, m_{n} \in$ $\mathcal{M}_{r}$ are extremal, rigid tree measures such that $\Sigma_{m_{i}}\left(m_{j}\right)=0$ for $i<j$. For every $p_{1}, p_{2}, \ldots, p_{n}>0$, the measure $m=\sum_{j=1}^{n} p_{j} m_{j}$ is rigid.

Proof The remarks above show that the first assertion is simply a reformulation of [1, Theorem 3.8]. For the converse, we proceed by induction, observing that the result is trivial for $n=1$. For the inductive step, the hypothesis implies $\Sigma_{m_{1}}(m)=-p_{1}$, and therefore $c_{m}=c_{m-p_{1} m_{1}}$ by Corollary 4.3.

\section{Mending fractured immersions}

We will analyze in more detail the main result of the preceding section. This analysis is a necessary preliminary for the results in Sect. 6. Let us fix a tree $T$ and an immersion $\varphi$ of $T$ which maps all the triple vertices of $T$ to $\triangle_{r}$. Let $m^{\prime}=m_{\varphi}$ be the corresponding measure in $\mathcal{M}_{r}$, and let $\mu \in \mathcal{M}_{r}$ be another measure. Fix for the moment an edge $e_{0}$ in $T$, and orient all the other edges of $T$ away from $e_{0}$. We define for every vertex $V$ of $T$ a number $\delta_{e_{0}}(V, \mu)$. Assume first that $V$ has order 2 and the corresponding edges are $A V, V B$, oriented toward $B$. Setting $A^{\prime}=\varphi(A), V^{\prime}=\varphi(V), B^{\prime}=\varphi(B)$, we set

$$
\delta_{e_{0}}(V, \mu)=\mu\left(V^{\prime} X\right),
$$

where $X$ is on the right side of $A^{\prime} V^{\prime}$, and $\varangle X V^{\prime} B^{\prime}=60^{\circ}$. On the other hand, if $V$ has order 3 and the corresponding edges are $A V, B V, C V$, with $A V$ oriented toward $V$, then

$$
\delta_{e_{0}}(V, \mu)=\mu\left(V^{\prime} X\right)
$$

where $A^{\prime}=\varphi(A), V^{\prime}=\varphi(V)$, and $X$ are collinear. When $V$ is one of the endpoints of $e_{0}$, we orient $e_{0}$ toward that endpoint in this definition. The following figure illustrates the two cases in the definition of $\delta_{e_{0}}(V, \mu)$. Some of the dotted edges may have 
positive density, but they do not enter in the definition of $\delta_{e_{0}}$.

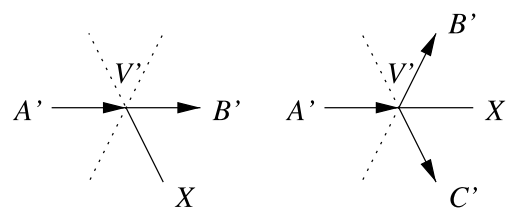

Theorem 4.2 can now be given a more precise form.

Theorem 5.1 With $\mu$ and $m^{\prime}$ as above, we have

$$
\Sigma_{m^{\prime}}(\mu)+\mu\left(\varphi\left(e_{0}\right)\right)=\sum_{V} \delta_{e_{0}}(V, \mu) .
$$

Proof The easiest way to see this is to cut $e_{0}$ in half, and orient the two halves away from its midpoint $Y$. Construct a fractured immersion $\psi$ of $T$ as in the proof of Theorem 4.2. For this immersion we have $\delta_{\psi}(V)=\delta_{e_{0}}(V, m)$ for each $V$, and $\delta_{g}(Y)=-\mu\left(\varphi\left(e_{0}\right)\right)$.

In the preceding proof, when $\varphi\left(e_{0}\right)$ is not contained in the support of $\mu$, the edge $\varphi\left(e_{0}\right)$ is simply translated along with the white puzzle piece which contains it. For our next result, it will be important that $m^{\prime}$ be a rigid measure and $\varphi\left(e_{0}\right)$ be a root edge for the measure $m^{\prime}$ with $m^{\prime}\left(\varphi\left(e_{0}\right)\right)=1$. With this choice, Lemma 3.2 implies the equality

$$
\delta_{e_{0}}\left(V, m^{\prime}\right)=0
$$

for every vertex $V$.

Let $T$ be a tree, and let $A V, V B$ be two edges meeting at a vertex $V$ of order 2 . One can stretch the tree to a tree $T^{\prime}$ replacing $V$ by a path $V_{1} V_{2} \cdots V_{k}$ of consecutive vertices of order 2 and the edges $A V$ and $B V$ are replaced by $A V_{1}$ and $B V_{k}$. Analogously, if $A V, B V, C V$ are three edges meeting at $V$, we can stretch $T$ by replacing $V$ with a 'tripod' formed by edges $V_{1} V_{2} \cdots V_{i} X, W_{1} W_{2} \cdots W_{j} X, U_{1} U_{2} \cdots U_{k} X$, where all new vertices except $X$ have order 2, and $A V, B V, C V$ are replaced by $A V_{1}, B W_{1}, C U_{1}$. If $T^{\prime}$ is obtained from $T$ by a finite number of such stretch operations, we will say that $T^{\prime}$ is a stretch of $T$. If $\varphi$ is an immersion of a stretch $T^{\prime}$ of $T$, the restriction of $\varphi$ to the original edges of $T$ determines a fractured immersion $\psi$ of $T$ with the property that $\delta_{\psi}(V)=0$ for every vertex $V$ of $T$. Such a fractured immersion of $T$ will be said to be stretchable. If $\psi$ is a stretchable fractured immersion and it is obtained as the restriction of an immersion $\varphi$, we will also write $m_{\psi}$ for the measure $m_{\varphi}$. The condition $\delta_{\psi}(V)=0$ for all $V$ is not sufficient for stretchability. For instance, assume that $V$ has degree 2, $A V, V B$ are the two adjacent edges mapped by $\psi$ to $A^{\prime} V^{\prime}$ and $V^{\prime \prime} B^{\prime}$. The condition $\delta_{\psi}(V)=0$ implies that the points $A^{\prime}, B^{\prime}, V^{\prime}, V^{\prime \prime}$ are collinear, but stretchability requires that $V^{\prime}$ and $V^{\prime \prime}$ should be between $A^{\prime}$ and $B^{\prime}$; the distance from $V^{\prime}$ to $V^{\prime \prime}$ is precisely the number of additional edges one must add at the point $V$. Similarly, if $A V, B V, C V$ are mapped to $A^{\prime} V^{\prime}, B^{\prime} V^{\prime \prime}, C^{\prime} V^{\prime \prime \prime}$, the 
condition $\delta_{\psi}(V)=0$ implies that these three lines intersect in a point $Z$, and stretchability requires that $V^{\prime}$ (resp. $V^{\prime \prime}, V^{\prime \prime \prime}$ ) be between $A^{\prime}$ (resp. $B^{\prime}, C^{\prime}$ ) and $Z$.

Part of the following argument (namely, the case $q=0$ ) amounts to a simplified proof of [1, Theorem 4.3].

Theorem 5.2 Let $\mu, m^{\prime} \in \mathcal{M}_{r}$, where $m^{\prime}$ is an extremal rigid measure assigning unit density to its root edges; in particular $m^{\prime}=m_{\varphi}$ for some immersion $\varphi$ of a tree $T$. Assume further that $\Sigma_{m^{\prime}}(\mu)=0$. Denote by $\alpha_{\ell}, \beta_{\ell}, \gamma_{\ell}$ and $\alpha_{\ell}^{\prime}, \beta_{\ell}^{\prime}, \gamma_{\ell}^{\prime}$ the exit densities of $\mu$ and $m^{\prime}$, respectively. There exists a stretchable fractured immersion $\psi$ of $T$ with the following properties.

(1) All the limits of $\psi$ at discontinuity points are contained in $\triangle_{r+\omega}(\mu)$.

(2) The exit densities $\widetilde{\alpha}_{i}^{\prime}$ of the corresponding measure $\tilde{m}^{\prime}=m_{\psi} \in \mathcal{M}_{r+\omega(\mu)}$ are only different from zero for $i=\ell+\sum_{s=0}^{\ell-1} \alpha_{s}, \ell=1,2, \ldots, r$, in which case $\widetilde{\alpha}_{i}^{\prime}=\alpha_{\ell}^{\prime}$, with similar formulas for $\widetilde{\beta}_{i}^{\prime}$ and $\widetilde{\gamma}_{i}^{\prime}$.

(3) The support of $m_{\psi}$ only crosses the dark gray parallelograms in the puzzle of $\mu$ along segments parallel to their edges.

Proof Denote by $q$ the largest integer with the property that $q m^{\prime} \leq \mu$. Assume first that $q=0$, in which case it is clear that the support of $m^{\prime}$ is not contained in the support of $\mu$. Choose an edge $e_{0}$ such that $\varphi\left(e_{0}\right)$ is contained in $\triangle_{r}$ and $\mu\left(\varphi\left(e_{0}\right)\right)=0$. Theorem 5.1 implies that $\delta_{e_{0}}(V, \mu)=0$ for every vertex $V$ of $T$. Orient all the edges of $T$ away from $e_{0}$, and construct a fractured immersion $\psi$ of $T$ by attaching each $\varphi(e)$ to the white puzzle piece of $\mu$ on its right. The condition $\delta_{e_{0}}(V)=0$ ensures that $\psi$ is stretchable at $V$, so that (1) holds. Since all the ends of $T$ are oriented outward, condition (2) is satisfied as well. The support of $m_{\psi}$ intersects the dark gray parallelograms along their edges, and along segments parallel to their light gray sides, as illustrated below. The oriented edges are in the support of $m^{\prime}$, and they, along with the dashed ones, may belong to $\operatorname{supp}(\mu)$. The figure degenerates when the support of $\mu$ is smaller, so that the dotted portions of the support of $m_{\psi}$ may be in the interior of a dark gray parallelogram.
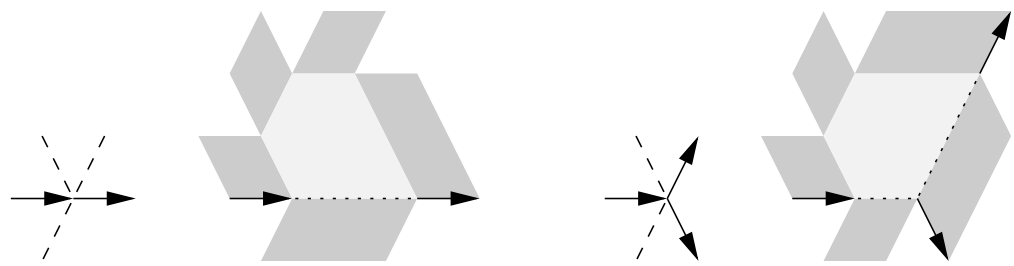

Consider now the case $q>0$, fix an edge $e_{0}$ such that $\varphi\left(e_{0}\right)$ is a root edge of $m^{\prime}$ contained in $\triangle_{r}$, and orient all the edges away from $e_{0}$. Give $e_{0}$ either orientation, and construct a fractured immersion $\psi_{0}$ of $T$ by attaching each $\varphi(e)$ to the white puzzle piece of $\mu$ on its right. To conclude the proof, it will suffice to construct a stretchable fractured immersion $\psi$ which coincides with $\psi_{0}$ on the ends of $T$. Note that $\psi_{0}(e)$ 
is now an edge of a dark gray parallelogram whose other side has length $\mu(e)$. We construct $\psi(e)$ by moving $\psi_{0}(e)$ inside this parallelogram a number of units equal to

$$
\sum_{V \geq e} \delta_{e_{0}}(V, \mu)
$$

away from the white piece to which $\psi_{0}(e)$ was attached, where the sum is extended over the vertices $V$ which are descendants of $e$ in the chosen orientation. In other words, the sum is extended over those vertices $V$ for which the shortest path from $e_{0}$ to $V$ passes through $e$. It is important to note that $\psi(e)$ really is contained in this (closed) gray parallelogram, and for this purpose it suffices to show that

$$
\sum_{V \geq e} \delta_{e_{0}}(V, \mu) \leq \mu(e)
$$

This follows from the fact that $\delta_{e_{0}}(V, \mu)=\delta_{e}(V, \mu)$ if $V \geq e$, and therefore

$$
\sum_{V \geq e} \delta_{e_{0}}(V, \mu)=\sum_{V \geq e} \delta_{e}(V, \mu) \leq \sum_{V} \delta_{e}(V, \mu)=\mu(e)
$$

by Theorem 5.1, since $\Sigma_{m^{\prime}}(\mu)=0$. Also observe that the position of $\psi\left(e_{0}\right)$ does not depend on the orientation chosen for $e_{0}$ because (with either orientation)

$$
\sum_{V \geq e_{0}} \delta_{e_{0}}(V, \mu)+\sum_{V \leq e_{0}} \delta_{e_{0}}(V, \mu)=\sum_{V} \delta_{e_{0}}(V, \mu)=\mu\left(e_{0}\right),
$$

and this is precisely the width of the dark gray parallelogram of which $\psi_{0}\left(e_{0}\right)$ is a side. It remains now to verify that $\psi$ is stretchable. Consider first two edges $e_{1}=A B, e_{2}=B C$ adjacent to a vertex $B$ of order 2 , oriented toward $B$ and $C$ respectively. Assume that $\varphi\left(e_{1}\right)=A^{\prime} B^{\prime}, \varphi\left(e_{2}\right)=B^{\prime} C^{\prime}$, and the small edge $B^{\prime} X$ is on the right of $A^{\prime} B^{\prime}$ such that $\varangle X B^{\prime} C^{\prime}=60^{\circ}$. We have then

$$
\sum_{V \geq e} \delta_{e_{0}}(V, \mu)=\mu\left(B^{\prime} X\right)+\sum_{V \geq f} \delta_{e_{0}}(V, \mu)
$$

so that $\psi_{0}\left(e_{1}\right)$ must be moved left $\mu\left(B^{\prime} X\right)$ more units than $\psi_{0}\left(e_{1}\right)$. This is precisely what is needed to align $\psi(e)$ and $\psi(f)$, as illustrated in the figure below, where the solid lines represent $\psi_{0}\left(e_{1}\right)$ and $\psi_{0}\left(e_{2}\right)$, the dashed lines represent $\psi(e)$ and $\psi(f)$, 
and the dotted line represents the range of the stretch of $\psi$.

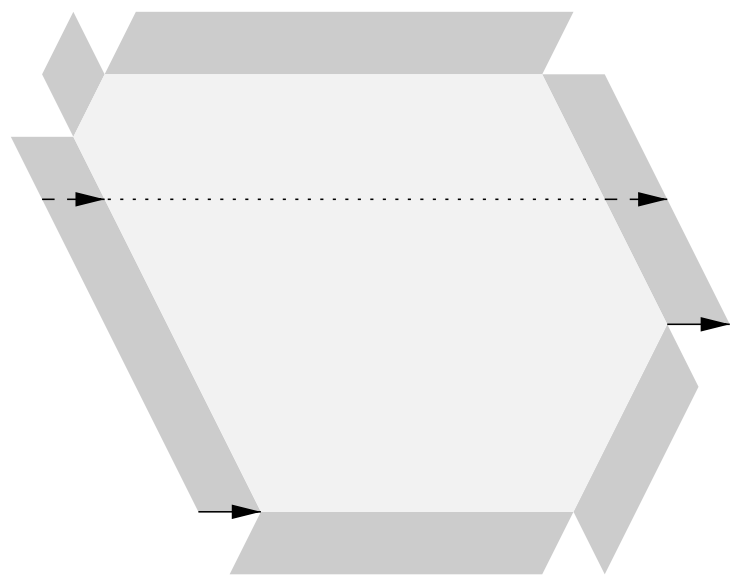

Assume now that $e_{1}=A V, e_{2}=B V, e_{3}=C V$ are three edges adjacent to $V$, such that $e_{1}$ is oriented toward $V$. These edges are mapped by $\varphi$ to $A^{\prime} V^{\prime}, B^{\prime} V^{\prime}, C^{\prime} V^{\prime}$. Let $V^{\prime} X$ be the small edge opposite $A^{\prime} V^{\prime}$. We have

$$
\sum_{V \geq e_{1}} \delta_{e_{0}}(V, \mu)=\mu\left(V^{\prime} X\right)+\sum_{V \geq e_{2}} \delta_{e_{0}}(V, \mu)+\sum_{V \geq e_{3}} \delta_{e_{0}}(V, \mu) .
$$

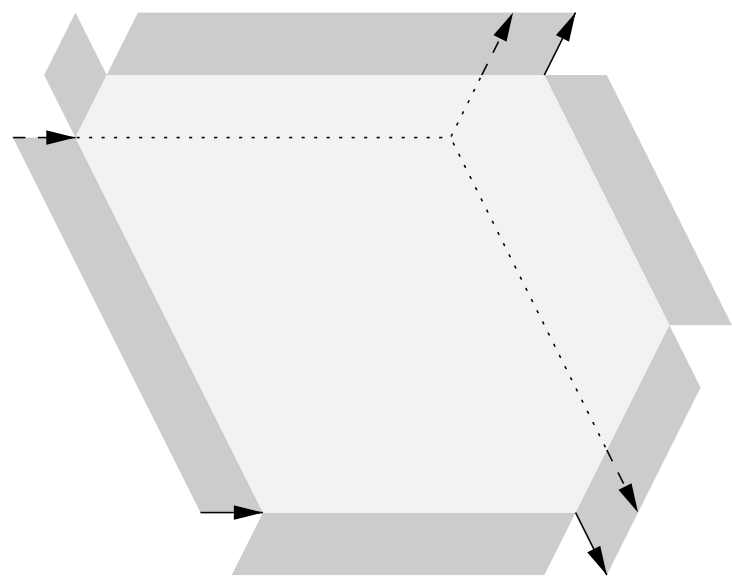

This relation is precisely what is needed to ensure that the break of $\psi$ at $V$ is stretchable, as in the illustration.

Remark 5.3 The preceding theorem produces a measure $\tilde{m}^{\prime}=m_{\psi}$ which is again a rigid tree measure. Indeed, $\widetilde{m}^{\prime}$ has the same nonzero densities as $m^{\prime}$, and therefore $\Sigma_{\widetilde{m}^{\prime}}\left(\widetilde{m}^{\prime}\right)=\Sigma_{m^{\prime}}\left(m^{\prime}\right)=-1$. In fact, it is easy to see that $\widetilde{m}^{\prime}$ is homologous to $m^{\prime}$. Indeed, using the notation in the proof above, this follows because two edges $e, e^{\prime}$ of 
$T$ such that $\varphi(e)=\varphi\left(e^{\prime}\right)$ will satisfy

$$
\sum_{V \geq e} \delta_{e_{0}}(V, m)=\sum_{V \geq e^{\prime}} \delta_{e_{0}}(V, m),
$$

and therefore their translates $\psi(e)$ and $\psi\left(e^{\prime}\right)$ will coincide as well.

Remark 5.4 The preceding remark extends easily to the more general case where $m^{\prime}=a_{1} m_{1}+a_{2} m_{2}+\cdots+a_{N} m_{N}$, where each $m_{j}$ is a rigid tree measure such that $\Sigma_{m_{j}}(\mu)=0$. Indeed, one simply applies the theorem to each $m_{j}$, and sets $\tilde{m}^{\prime}=$ $a_{1} \widetilde{m}_{1}+a_{2} \widetilde{m}_{2}+\cdots+a_{N} \widetilde{m}_{N}$. If the measure $m^{\prime}$ is rigid, then $\widetilde{m}^{\prime}$ is rigid as well, and homologous to $m^{\prime}$.

Remark 5.5 Theorem 5.2 is true for arbitrary tree measures as well. However, the stretched measure $\tilde{m}^{\prime}$ is not generally homologous to $m^{\prime}$. The reason is that (5.1) may fail, and therefore two edges $\varphi(e)=\varphi\left(e^{\prime}\right)$ may be translated by different amounts in the puzzle of $\mu$. The proof needs almost no alteration. However, since the concept of a root edge is not defined for non-rigid measures, we choose $e_{0}$ to be any edge such that $\varphi\left(e_{0}\right) \subset \triangle_{r}$.

\section{Reduction of the intersection problem}

We are now ready to discuss the reduction procedures mentioned in the introduction. We recall first some facts from [1]. Fix a measure $m \in \mathcal{M}_{r}$ with integer densities. A point $A_{\ell}$ (resp. $B_{\ell}, C_{\ell}$ ) is called an attachment point of $m$ if $\ell \geq 1$ and $m\left(A_{\ell} X_{\ell}\right)>0$ (resp. $\left.m\left(B_{\ell} Y_{\ell}\right)>0, m\left(C_{\ell} Z_{\ell}\right)>0\right)$. We denote by att $(m)$ (resp. $\left.\operatorname{att}_{J}(m), \operatorname{att}_{K}(m)\right)$ the collection of indices $\ell \in\{1,2, \ldots, r\}$ such that $A_{\ell}$ (resp. $\left.B_{\ell}, C_{\ell}\right)$ is an attachment point for $m$.

Let now $I_{m}, J_{m}, K_{m} \subset\{1,2, \ldots, n=r+\omega(m)\}$ be the sets of cardinality $r$ defined by (2.2). The index $i_{\ell} \in I_{m}$ (resp. $j_{\ell} \in J_{m}, k_{\ell} \in K_{m}$ ) is called an attachment index for $m$ if $A_{\ell}$ (resp. $B_{\ell}, C_{\ell}$ ) is an attachment point. We denote by $I_{m}^{\text {att }}, J_{m}^{\text {att }}, K_{m}^{\text {att }}$ the collections of attachment indices; thus $I_{m}^{\text {att }}=\left\{i_{\ell}: \ell \in \operatorname{att}_{I}(m)\right\}$. Assume further that we are given flags $\mathcal{E}, \mathcal{F}, \mathcal{G}$ in $\mathbb{C}^{n}$. The spaces $\left\{\mathbb{E}_{i_{\ell}}: \ell \in I_{m}^{\text {att }}\right\},\left\{\mathbb{F}_{j_{\ell}}: \ell \in J_{m}^{\text {att }}\right\},\left\{\mathbb{G}_{k_{\ell}}\right.$ : $\left.\ell \in K_{m}^{\text {att }}\right\}$ are called the attachment spaces of $m$.

Let now $\tilde{m} \in \mathcal{M}_{\widetilde{r}}$ be a second measure with integer densities. If $m$ and $\tilde{m}$ are homologous, there clearly exist order preserving bijections $\varphi_{I}: I_{m}^{\text {att }} \rightarrow I_{\widetilde{m}}^{\text {att }}, \varphi_{J}: J_{m}^{\text {att }} \rightarrow$ $J_{\widetilde{m}}^{\text {att }}, \varphi_{K}: K_{m}^{\text {att }} \rightarrow K_{\widetilde{m}}^{\text {att }}$.

Also recall that a lattice polynomial of a collection $\mathcal{X}=\left(\mathbb{X}_{\nu}\right)_{v \in N}$ of spaces is defined inductively by the requirements that

(1) for each $v$, the expression $P_{v}(\mathcal{X})=\mathbb{X}_{v}$ is a lattice polynomial, and

(2) if $P(\mathcal{X})$ and $Q(\mathcal{X})$ are lattice polynomials, then $(P(\mathcal{X}))+(Q(\mathcal{X}))$ and $(P(\mathcal{X})) \cap$ $(Q(\mathcal{X}))$ are also lattice polynomials.

More formally, lattice polynomials should be defined as elements of an abstract lattice generated by a set of variables indexed by $N$. One can then substitute subspaces for 
the variables to obtain a new subspace. This gives the proper meaning to the last statement in the next theorem.

The following result is a reformulation of results in [1]. The fact that the lattice polynomial is essentially the same for all homologous measures is not explicitly stated there, but it is easily verified using the argument of [1, Proposition 5.1].

Theorem 6.1 Assume that $m \in \mathcal{M}_{r}$ is a rigid measure with integer densities, and $\mathcal{E}, \mathcal{F}, \mathcal{G}$ are flags in $\mathbb{C}^{n}, n=r+\omega(m)$. There exists a lattice polynomial $P_{m}$ of the attachment spaces of $m$ such that generically

$$
P_{m}\left(\mathbb{E}_{i}, \mathbb{F}_{j}, \mathbb{G}_{k}: i \in I_{m}^{\mathrm{att}}, j \in J_{m}^{\mathrm{att}}, k \in K_{m}^{\mathrm{att}}\right) \in \mathfrak{S}\left(\mathcal{E}, I_{m}\right) \cap \mathfrak{S}\left(\mathcal{F}, J_{m}\right) \cap \mathfrak{S}\left(\mathcal{G}, K_{m}\right) .
$$

Moreover, if $\tilde{m} \in \mathcal{M}_{\tilde{r}}$ is homologous to $m$ and $\widetilde{\mathcal{E}}, \widetilde{\mathcal{F}}, \widetilde{\mathcal{G}}$ are flags in $\mathbb{C}^{\tilde{n}}$,

$$
P_{\widetilde{m}}\left(\widetilde{\mathbb{E}}_{\varphi_{I}(i)}, \widetilde{\mathbb{F}}_{\varphi_{J}(j)}, \widetilde{\mathbb{G}}_{\varphi_{K}(k)}: i \in I_{m}^{\mathrm{att}}, j \in J_{m}^{\mathrm{att}}, k \in K_{m}^{\mathrm{att}}\right)
$$

equals

$$
P_{m}\left(\widetilde{\mathbb{E}}_{\varphi_{I}(i)}, \widetilde{\mathbb{F}}_{\varphi_{J}(j)}, \widetilde{\mathbb{G}}_{\varphi_{K}(k)}: i \in I_{m}^{\mathrm{att}}, j \in J_{m}^{\mathrm{att}}, k \in K_{m}^{\mathrm{att}}\right) .
$$

Given a measure (rigid or not) $m \in \mathcal{M}_{r}$ and an extremal, rigid tree measure $m^{\prime} \in \mathcal{M}_{r}$, we will be able to apply a reduction of the Schubert problem associated to $m$ provided that $\Sigma_{m^{\prime}}(m)=-p<0$. More precisely, the Schubert problem will be reduced to the corresponding problem for the measure $m-p m^{\prime}$ (which satisfies $c_{m-p m^{\prime}}=c_{m}$ by Corollary 4.3) in a space $\mathbb{X}$ of dimension $n-p \omega\left(m^{\prime}\right)$. The space $\mathbb{X}$ is obtained by applying the lattice polynomial $P_{m^{\prime}}$ to the attachment spaces of $m$ corresponding to the attachment points of $m^{\prime}$. The following result describes the procedure in detail. The argument is essentially contained in [1, Proposition 5.1], but we include it here for completeness, and as a practical recipe. Observe that $\Sigma_{m^{\prime}}(m)+\omega^{\prime} n$ is precisely the sum (1.2) mentioned in our initial discussion of reductions.

For the following result, recall that rigid tree measures $m^{\prime}$ assign unit mass to their root edges, and hence $\Sigma_{m^{\prime}}\left(m^{\prime}\right)=-1$ by Theorem $4.2(3)$.

Theorem 6.2 Let $m, m^{\prime} \in \mathcal{M}_{r}$ be two measures with integer densities such that $m^{\prime}$ $i$ a rigid tree measure, and $\Sigma_{m^{\prime}}(m)=-p<0$. Denote by $i_{\ell}, j_{\ell}, k_{\ell}, \ell=1,2, \ldots, r$, the elements of $I=I_{m}, J=J_{m}, K=K_{m}$, respectively. Given generic flags $\mathcal{E}, \mathcal{F}, \mathcal{G}$ in $\mathbb{C}^{n}, n=r+\omega(m)$, the space

$$
\mathbb{X}=P_{m^{\prime}}\left(\mathbb{E}_{i_{x}}, \mathbb{F}_{j_{y}}, \mathbb{G}_{k_{z}}: x \in \operatorname{att}_{I}\left(m^{\prime}\right), y \in \operatorname{att}_{J}\left(m^{\prime}\right), z \in \operatorname{att}_{K}\left(m^{\prime}\right)\right)
$$

has dimension $n-p \omega\left(m^{\prime}\right)$. Moreover, denote by $\mathcal{E}^{\prime}$ the flag in $\mathbb{X}$ obtained by intersecting the spaces in $\mathcal{E}$ with $\mathbb{X}$ and discarding repeating spaces, with similar definitions for $\mathcal{F}^{\prime}$ and $\mathcal{G}^{\prime}$. Then we have

$$
\begin{aligned}
& \mathfrak{S}\left(\mathcal{E}^{\prime}, I_{m-p m^{\prime}}\right) \cap \mathfrak{S}\left(\mathcal{F}^{\prime}, J_{m-p m^{\prime}}\right) \cap \mathfrak{S}\left(\mathcal{G}^{\prime}, K_{m-p m^{\prime}}\right) \\
& \quad \subset \mathfrak{S}\left(\mathcal{E}, I_{m}\right) \cap \mathfrak{S}\left(\mathcal{F}, J_{m}\right) \cap \mathfrak{S}\left(\mathcal{G}, K_{m}\right) .
\end{aligned}
$$


Proof Denote the exit densities of $m$ by $a_{\ell}, b_{\ell}, c_{\ell}, \ell=0,1, \ldots, r$. Thus the elements of $I_{m}, J_{m}, K_{m}$ are given by

$$
i_{\ell}=\ell+\sum_{\ell^{\prime}<\ell} a_{\ell^{\prime}}, \quad j_{\ell}=\ell+\sum_{\ell^{\prime}<\ell} b_{\ell^{\prime}}, \quad k_{\ell}=\ell+\sum_{\ell^{\prime}<\ell} c_{\ell^{\prime}}
$$

for $\ell=1,2, \ldots, r$. By Corollary 4.3, we can write $m=p m^{\prime}+\mu$, where $\mu \in \mathcal{M}_{r}$, and $\Sigma_{m^{\prime}}(\mu)=0$. Denote the exit densities of $\mu$ and $m^{\prime}$ by $\alpha_{\ell}, \beta_{\ell}, \gamma_{\ell}$ and $\alpha_{\ell}^{\prime}, \beta_{\ell}^{\prime}, \gamma_{\ell}^{\prime}$, respectively. We have

$$
a_{\ell}=\alpha_{\ell}+p \alpha_{\ell}^{\prime}, \quad b_{\ell}=\beta_{\ell}+p \beta_{\ell}^{\prime}, \quad c_{\ell}=\gamma_{\ell}+p \gamma_{\ell}^{\prime}
$$

for $\ell=0,1, \ldots, r$. Theorem 5.2 yields a rigid tree measure $\tilde{m}^{\prime} \in \mathcal{M}_{r+\omega(\mu)}$, homologous to $m^{\prime}$, whose only possible nonzero exit densities are $\widetilde{\alpha}_{i}^{\prime}=\alpha_{\ell}^{\prime}$ for

$$
i=\ell+\sum_{k<\ell} \alpha_{k}=\ell+\sum_{k<\ell}\left(a_{k}-p \alpha_{k}^{\prime}\right), \quad \ell=0,1,2, \ldots, r
$$

with analogous formulas for $\widetilde{\beta}_{i}^{\prime}$ and $\tilde{\gamma}_{i}^{\prime}$. The set $I_{p \tilde{m}^{\prime}}$ of cardinality $r+\omega(\mu)$ has elements

$$
\widetilde{i}_{x}=x+\sum_{y<x} p \widetilde{\alpha}_{y}^{\prime}, \quad x=1,2, \ldots, r+\omega(\mu) .
$$

In particular

$$
\tilde{i}_{x}=i_{\ell} \quad \text { when } x=\ell+\sum_{k<\ell} \alpha_{k}
$$

with similar formulas for $\widetilde{j}_{x}$ and $\widetilde{k}_{x}$. We deduce that the attachment spaces of $p \tilde{m}^{\prime}$ are precisely

$$
\left\{\mathbb{E}_{i_{x}}, \mathbb{F}_{j_{y}}, \mathbb{G}_{k_{z}}: x \in \operatorname{att}_{I}\left(m^{\prime}\right), y \in \operatorname{att}_{J}\left(m^{\prime}\right) z \in \operatorname{att}_{K}\left(m^{\prime}\right)\right\}
$$

Now, the measure $p \tilde{m}^{\prime}$ is homologous to $m^{\prime}$, and therefore Theorem 6.1 implies that the space $\mathbb{X}$ in our statement belongs generically to the intersection

$$
\mathfrak{S}\left(\mathcal{E}, I_{p \tilde{m}^{\prime}}\right) \cap \mathfrak{S}\left(\mathcal{F}, J_{p \tilde{m}^{\prime}}\right) \cap \mathfrak{S}\left(\mathcal{G}, K_{p \tilde{m}^{\prime}}\right)
$$

Relation (6.1) implies that

$$
\operatorname{dim}\left(\mathbb{X} \cap \mathbb{E}_{i_{\ell}}\right) \geq \ell+\sum_{k<\ell} \alpha_{k}, \quad \ell=1,2, \ldots, r,
$$

with similar estimates for $\operatorname{dim}\left(\mathbb{X} \cap \mathbb{F}_{j_{\ell}}\right)$ and $\operatorname{dim}\left(\mathbb{X} \cap \mathbb{G}_{k_{\ell}}\right)$. Thus, by intersecting the spaces in the flags $\mathcal{E}, \mathcal{F}, \mathcal{G}$ with $\mathbb{X}$ we obtain (after eliminating repeating spaces) flags $\mathcal{E}^{\prime}, \mathcal{F}^{\prime}, \mathcal{G}^{\prime}$ in $\mathbb{X}$ with the property that

$$
\mathbb{E}_{x}^{\prime} \subset \mathbb{X} \cap \mathbb{E}_{i_{\ell}} \text { for } x=\ell+\sum_{k<\ell} \alpha_{k},
$$


and similarly for $\mathcal{F}^{\prime}$ and $\mathcal{G}^{\prime}$.

Note now that $\omega\left(m-p m^{\prime}\right)=n-p \omega\left(m^{\prime}\right)=\operatorname{dim}(\mathbb{X})$, and therefore it makes sense to solve the Schubert problem associated with this measure and the flags $\mathcal{E}^{\prime}, \mathcal{F}^{\prime}, \mathcal{G}^{\prime}$. To conclude the proof, let $\mathbb{M}$ be a space in the intersection

$$
\mathfrak{S}\left(\mathcal{E}^{\prime}, I_{m-p m^{\prime}}\right) \cap \mathfrak{S}\left(\mathcal{F}^{\prime}, J_{m-p m^{\prime}}\right) \cap \mathfrak{S}\left(\mathcal{G}^{\prime}, K_{m-p m^{\prime}}\right) .
$$

To see that $\mathbb{M}$ belongs to

$$
\mathfrak{S}\left(\mathcal{E}, I_{m}\right) \cap \mathfrak{S}\left(\mathcal{F}, J_{m}\right) \cap \mathfrak{S}\left(\mathcal{G}, K_{m}\right),
$$

observe that the $\ell$ th element of $I_{m-p m^{\prime}}$ is equal to $i=\ell+\sum_{k<\ell} \alpha_{k}$, so that

$$
\operatorname{dim}\left(\mathbb{M} \cap \mathbb{E}_{i_{\ell}}\right)=\operatorname{dim}\left(\mathbb{M} \cap\left(\mathbb{X} \cap \mathbb{E}_{i_{\ell}}\right)\right) \geq \operatorname{dim}\left(\mathbb{M} \cap \mathbb{E}_{i}^{\prime}\right) \geq \ell,
$$

where we used (6.2) in the first inequality.

When the measure $m$ is itself rigid, we can represent it as in Corollary 4.5. That is, there exist rigid tree measures $m_{1}, m_{2}, \ldots, m_{n}$ and positive integers $p_{1}, p_{2}, \ldots, p_{n}$ such that $m=\sum_{i=1}^{n} p_{i} m_{i}$, and $\Sigma_{m_{1}}\left(m_{i}\right)=0$ for $i=2,3, \ldots, n$. Choosing $m^{\prime}=m_{1}$, we have $\Sigma_{m^{\prime}}(m)=-p_{1}<0$, and $m-p_{1} m^{\prime}=\sum_{i=2}^{n} p_{i} m_{i}$ has fewer extremal summands than $m$. Repeating this procedure reduces the intersection problem associated to $m$ to the problems corresponding to the measures $m_{1}, m_{2}, \ldots, m_{n}$. Note, however, that this apparently very simple method assumes knowledge of the lattice polynomials $P_{m_{j}}$ for $j=1,2, \ldots, n$. The actual calculation of these polynomials is done recursively using the fact that, with rare exceptions, the dual $m^{*}$ of a rigid extremal measure is not extremal [1, Proposition 5.2].

As an illustration, we will see how to deduce the two kinds of reductions mentioned in the Introduction. First, consider a measure $m^{\prime}$ with $\omega\left(m^{\prime}\right)=1$. There are only three nonzero exit densities $\alpha_{x}^{\prime}=\beta_{y}^{\prime}=\gamma_{z}^{\prime}=1$, and we must have $x+y+z=r$; see the first triangle in the figure below. The sum

$$
\Sigma_{m^{\prime}}(\mu)=i_{x}+j_{y}+k_{z}-n
$$

corresponds to the original reductions in [9], and a reduction can be applied when this sum is negative. The relevant lattice polynomial is

$$
P_{m^{\prime}}(\mathbb{E}, \mathbb{F}, \mathbb{G})=\mathbb{E}+\mathbb{F}+\mathbb{G},
$$

thus yielding the formula mentioned in the introduction. The second reduction outlined in the introduction corresponds with a measure $m^{\prime}$ satisfying $\omega\left(m^{\prime}\right)=2$ whose support is shown in the second triangle below.
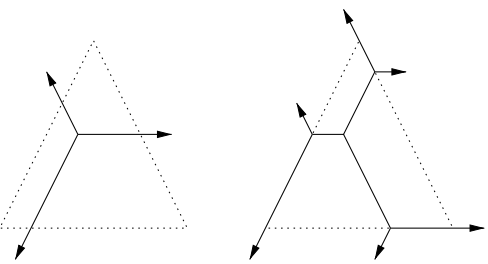
There are now six exit densities equal to 1 , but three of them are $\alpha_{0}^{\prime}, \beta_{0}^{\prime}, \gamma_{0}^{\prime}$, which do not correspond to attachment points. The others are $\alpha_{x}^{\prime}, \beta_{y}^{\prime}, \gamma_{z}^{\prime}$, where the numbers $x, y, z$ are the lengths of the dotted segments in the boundary of $\triangle_{r}$. Clearly $x+y+$ $z=2 r$, and

$$
\Sigma_{m^{\prime}}(\mu)=i_{x}+j_{y}+k_{z}-2 n .
$$

This time the lattice polynomial is

$$
P_{m^{\prime}}(\mathbb{E}, \mathbb{F}, \mathbb{G})=(\mathbb{E} \cap \mathbb{F})+(\mathbb{F} \cap \mathbb{G})+(\mathbb{G} \cap \mathbb{E}) .
$$

We conclude this section with an analysis of the measures in $\mathcal{M}_{3}$ which do not allow any of the reductions outlined above. There are 11 extremal measures in $\mathcal{M}_{3}$, and all of them are rigid tree measures. Their supports are depicted below.

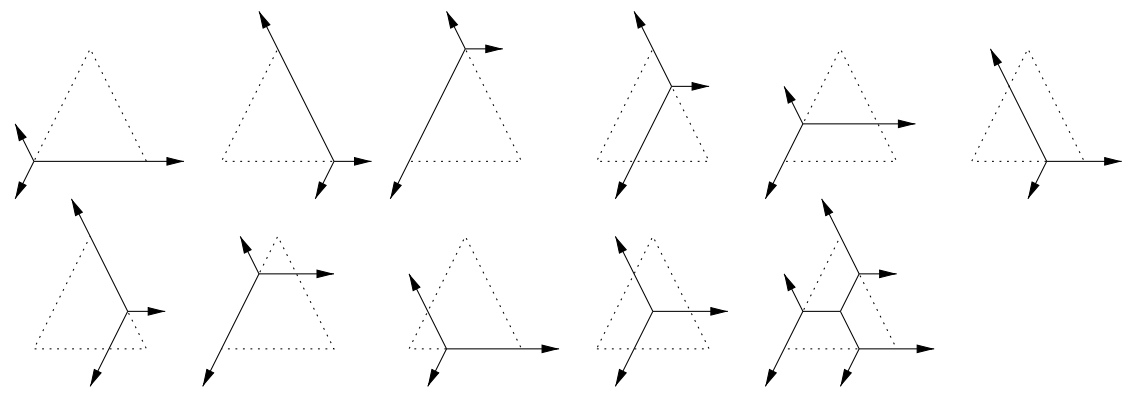

Let us call these tree measures $\mu_{1}, \mu_{2}, \mu_{3}, v_{1}, \nu_{2}, \nu_{3}, \rho_{1}, \rho_{2}, \rho_{3}, \tau_{1}$, and $\tau_{2}$. In addition to the equalities $\Sigma_{\mu}(\mu)=-1$, the only other nonzero values for $\Sigma_{\mu}(\nu)$ with $\mu, \nu$ among these measures are equal to one. These are: $\Sigma_{v_{j}}\left(\mu_{j}\right), \Sigma_{\rho_{j}}\left(\mu_{j}\right), \Sigma_{\tau_{1}}\left(\mu_{j}\right)$ for $j=1,2,3$, and the three cycles $\Sigma_{v_{j}}\left(v_{j+1}\right), \Sigma_{\rho_{j}}\left(\rho_{j-1}\right)$, and $\Sigma_{\tau_{j}}\left(\tau_{j+1}\right)$. An arbitrary measure $m \in \mathcal{M}_{3}$ with integer densities can be written as

$$
m=\sum_{j=1}^{3}\left(a_{j} \mu_{j}+b_{j} v_{j}+c_{j} \rho_{j}\right)+d_{1} \tau_{1}+d_{2} \tau_{2},
$$

where the coefficients $a_{j}, b_{j}, c_{j}, d_{j}$ are non-negative integers. Note that $\Sigma_{\mu_{j}}(m)=$ $-a_{j}, \Sigma_{v_{j}}(m)=a_{j}+b_{j+1}-b_{j}, \Sigma_{\rho_{j}}(m)=a_{j}+c_{j-1}-c_{j}, \Sigma_{\tau_{2}}(m)=d_{1}-d_{2}$, and $\Sigma_{\tau_{1}}(m)=a_{1}+a_{2}+a_{3}+d_{2}-d_{1}$. A reduction is possible unless $a_{1}=a_{2}=a_{3}=0$, $b_{1}=b_{2}=b_{3}, c_{1}=c_{2}=c_{3}$, and $d_{1}=d_{2}$. Observe also that $\tau_{1}+\tau_{2}=v_{1}+v_{2}+v_{3}$, and this measure has the same exit densities as $\rho_{1}+\rho_{2}+\rho_{3}$. Thus the only intersection problems which cannot be reduced with our methods arise from measures of the form $d\left(\tau_{1}+\tau_{2}\right)$ for some integer $d>0$. The measure $m$ is rigid if and only if $b_{1} b_{2} b_{3}=$ $c_{1} c_{2} c_{3}=d_{1} d_{2}=0$. The first ten of the rigid tree measures above correspond with the reductions considered in [9] and [3]. It should be noted that this analysis can be applied, via the duality described in [1], to the analysis of measures $m$ with $\omega(m)=3$. The intersection problems for such measures can be reduced to duals of measures of the form $d\left(\tau_{1}+\tau_{2}\right)$. 
A similar analysis can be carried out for $r=4$ and $r=5$, but with many more tree measures. Indeed, for $r \leq 5$ all extremal measures in $\mathcal{M}_{r}$ are rigid. For $r=6$ there are already some extremal measures which are not tree measures, though their exit densities coincide with those of a sum of extremal rigid measures. An example is provided below, where all solid edges have unit density. The two resulting measures have the same exit densities, but only the first one is extremal; the second one is the sum of either two or three extremal measures.
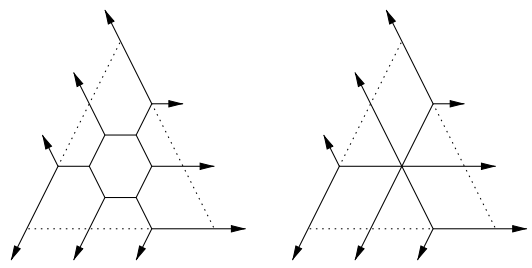

For larger values of $r$, there exist tree extremal measures which are not rigid, and do not have the same exit densities as any sum of extremal rigid measures. The support of such a tree measure $m$ is pictured below.

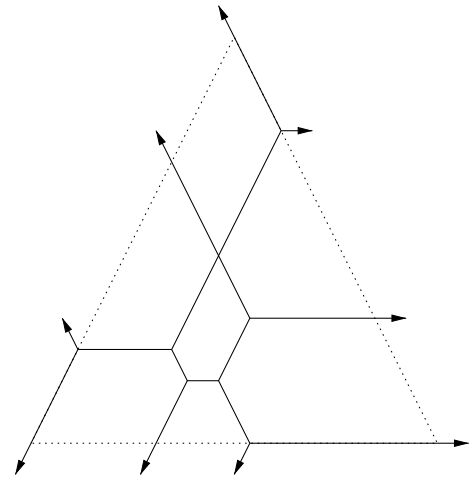

Here $r=13$, and the exit points are $A_{0}, A_{4}, A_{10}, B_{0}, B_{4}, B_{7}, C_{0}, C_{4}$, and $C_{10}$. It is easy to verify that one cannot find among these points $A_{x}, B_{y}, C_{z}$ such that $x+$ $y+z=13$, and therefore the exit densities do not majorize the exit densities of any measure $\mu$ with $\omega(\mu)=1$. Since $\omega(m)=3$, it follows that the exit densities of $m$ do not majorize those of any rigid tree measure.

\section{Connection with multiplication formulas}

Triples $(I, J, K)$ with the property that $c_{I J K}>0$ are characterized by the identity (1.1) and the Horn inequalities. The system of Horn inequalities is redundant, but it can be reduced to a non-redundant collection of essential Horn inequalities. It was proved in [5, Theorem 7.14] and [7, Theorem 1.4] that, when one of these essential inequalities is saturated (that is, it is an equality), the coefficient $c_{I J K}$ can be written as the product of two Littlewood-Richardson coefficients corresponding to smaller 
sets. The result in [5] is deduced from more general considerations about quiver representations, while the approach of [7] is closer to ours.

We begin here by formulating this result in terms of measures. Our formulation depends on seeing that the Horn inequalities can be deduced by superposing the puzzle of a measure over the support of another measure in $\mathcal{M}_{r}$. It was first observed in [4] that the Horn inequalities can be proved this way, and the formulation in terms of measures appears in [1]. Consider therefore a measure $m \in \mathcal{M}_{r}$ and a measure $v \in \mathcal{M}_{s}$ with the property that $s+\omega(v)=r$. The puzzle of $v$ sits in a triangle of size $r$ which we take to be precisely $\triangle_{r}$. As seen in [1], the puzzle of $v$ indicates a certain Horn inequality which is saturated precisely when the support of $m$ crosses the dark gray parallelograms of this puzzle on segments parallel to their edges. The inequality is essential precisely when $v$ is rigid. The following illustration shows an example when such a Horn inequality is saturated. The solid lines represent the support of $m$, and the densities can be taken to be 1 on the entire support.

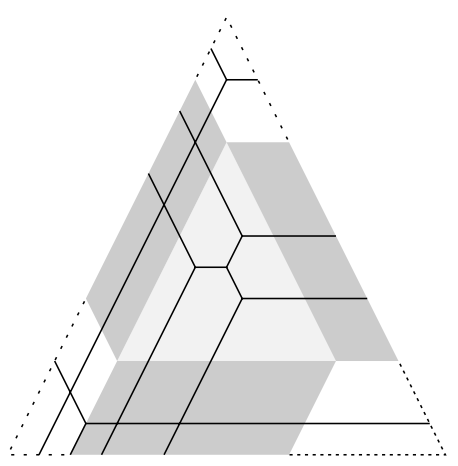

One can now construct two measures $m_{v} \in \mathcal{M}_{s}$ and $m_{v^{*}} \in \mathcal{M}_{\omega(v)}$ in the following way. To construct $m_{v}$, delete all light gray pieces and dark gray parallelograms, and move the white pieces along with the remaining support of $m$ together. This yields the measure $m_{v}$ provided that we also assign to each small edge $e$ on the border of a white piece a density equal to the total of the densities on the support of $m$ on lines crossing the corresponding dark gray parallelogram and parallel to $e$. The measure $m_{v^{*}}$ is obtained in an analogous manner by deleting the white puzzle pieces and the dark gray parallelograms. For the measures $m$ and $v$ illustrated above we obtain the measures in the next figure. The thicker lines indicate edges where the measure $m_{v}$ has densities 2 and 3 .

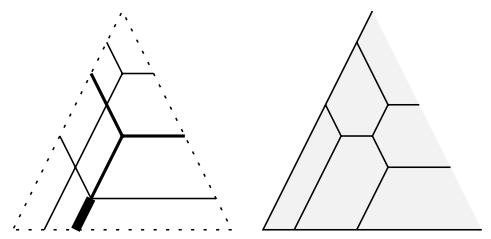

We collect in the following result a few useful facts about this construction. 
Lemma 7.1 Assume that a measure $m \in \mathcal{M}_{r}$ saturates the Horn inequality determined by the puzzle of a measure $v \in \mathcal{M}_{s}$ with $s+\omega(v)=r$. Then

(1) $\omega\left(m_{v}\right)=\omega\left(m_{v^{*}}\right)=\omega(m)$.

(2) The exit densities of $m_{v}$ and $m_{v^{*}}$ are determined by the exit densities of $m$ and by the puzzle of $v$.

(3) $\left(m_{v}\right)^{*}+\left(m_{v^{*}}\right)^{*}=m^{*}$.

Proof Parts (1) and (2) are obvious. To prove (3), we observe that the support of $\left(m_{v}\right)^{*}$ is contained in the support of $m^{*}$. More precisely, an edge $X Y$ in the support of $m^{*}$ has density equal to the length of the corresponding edge $A B$ in the support of $m$. The measure $\left(m_{v}\right)^{*}$ assigns $X Y$ a density equal to the length of the white portion of $A B$, that is, the sum of all the lengths of those parts of $A B$ contained in a white puzzle piece. Similarly, $\left(m_{v^{*}}\right)^{*}$ assigns $X Y$ a density equal to the light gray portion of $A B$; see the discussion of duality in Sect. 4. These considerations, suitably modified for segments $A B$ which contain edges of dark gray parallelograms, immediately yield (3).

Theorem 1.4 of [7] can now be formulated as follows.

Theorem 7.2 With the above notation, we have $c_{m} \leq c_{m_{v}} c_{m_{v^{*}}}$. If the measure $v$ is rigid, then $c_{m}=c_{m_{v}} c_{m_{v^{*}}}$.

Strict inequality can hold in this result, and equality may hold even when $v$ is not rigid, for instance when $c_{m_{v}}=c_{m_{v^{*}}}=1$. As an illustration, consider a measure $m \in \mathcal{M}_{6}$ whose only nonzero exit densities are

$$
\alpha_{0}=\alpha_{2}=\alpha_{4}=\beta_{0}=\beta_{2}=\beta_{4}=\gamma_{0}=\gamma_{2}=\gamma_{4}=s,
$$

where $s$ is a non-negative integer; the example given in [7] corresponds to $s=1$. The following figure represents the possible support of $m$ along with the puzzle of a measure $v$, and the supports of the measures $m_{v}$ and $m_{v^{*}}$. Both of these measures have exit densities

$$
\alpha_{0}=\alpha_{1}=\alpha_{2}=\beta_{0}=\beta_{1}=\beta_{2}=\gamma_{0}=\gamma_{1}=\gamma_{2}=s,
$$

and it is easy to calculate

$$
c_{m}=\left(\begin{array}{c}
s+2 \\
2
\end{array}\right), \quad c_{m_{v}}=c_{m_{v^{*}}}=s+1, \quad c_{m_{v}} c_{m_{v^{*}}}-c_{m}=\left(\begin{array}{c}
s+1 \\
2
\end{array}\right) .
$$

The inequality $c_{m} \leq c_{m_{v}} c_{m_{v^{*}}}$ is strict, except for the degenerate case $s=0$.
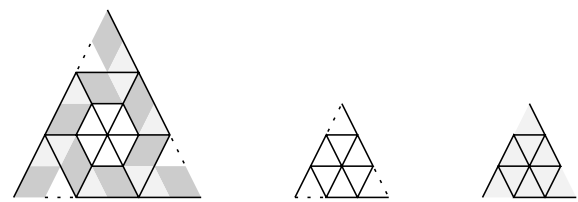
The first part of Theorem 7.2 is not actually stated in [7]. This is essentially a uniqueness statement, and it is fairly easy to deduce from the arguments of [7]: given the measures $m_{v}$ and $m_{v^{*}}$ and the exit densities of $m$, the measure $m$ is uniquely determined. Lemma 7.1(3) provides an easy proof, and it does not even require advance knowledge of the exit densities of $m$. The more difficult second part is an existence statement: given measures $\mu \in \mathcal{M}_{s}$ and $\mu_{*} \in \mathcal{M}_{\omega(v)}$ with the same exit densities as $m_{v}$ and $m_{v^{*}}$ respectively, there exists a measure $\rho \in \mathcal{M}_{r}$ with the same exit densities as $m$ such that $\rho_{v}=\mu$ and $\rho_{v^{*}}=\mu_{*}$. The obvious candidate is determined by the equation $\rho^{*}=\mu^{*}+\left(\mu_{*}\right)^{*}$.

We have $c_{m}=c_{m^{*}}$, where $m^{*} \in \mathcal{M}_{\omega(m)}^{*}$ is the dual of $m$. It follows that Theorem 7.2 also yields a factorization of $c_{m}$ when $m^{*}$ saturates a Horn inequality arising from a rigid puzzle. We show next that, under the conditions of Corollary 4.3, the measure $m^{*}$ does saturate such an inequality. The measures $\left(m^{*}\right)_{v}$ and $\left(m^{*}\right)_{v^{*}}$ in the following statement are the analogues of $m_{v}$ and $m_{v^{*}}$ for measures in $\mathcal{M}_{\omega(m)}^{*}$.

Theorem 7.3 Assume that $m, m^{\prime} \in \mathcal{M}_{r}, m^{\prime}$ is a rigid tree measure, and $\Sigma_{m^{\prime}}(m)=$ $-p<0$. Denote $\mu=m-p m^{\prime}$. There exists a rigid measure $v \in \mathcal{M}_{\omega(\mu)}$ with the following properties.

(1) $\omega(v)=p \omega\left(m^{\prime}\right)$ and the support of $v^{*}$ is contained in the support of $\left(\mathrm{pm}^{\prime}\right)^{*}$.

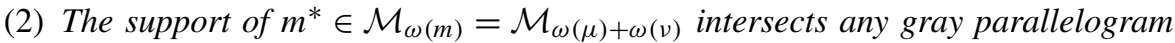
in the puzzle of $v$ only on line segments parallel to its sides. In particular, $m^{*}$ saturates the Horn inequality determined by the puzzle of $v$.

(3) $\left(m^{*}\right)_{v}=\mu^{*}$.

(4) The support of $\left(m^{*}\right)_{v^{*}}$ is contained in the support of $v^{*}$. In particular, $\left(m^{*}\right)_{v^{*}}$ is rigid.

Proof The equality $\Sigma_{m^{\prime}}(\mu)=\Sigma_{m^{\prime}}(m)-p \Sigma_{m^{\prime}}\left(m^{\prime}\right)=0$ allows us to apply Theorem 5.2 to obtain a tree measure $\rho=\tilde{m}^{\prime} \in \mathcal{M}_{r+\omega(\mu)}$, homologous with $m^{\prime}$, and with properties (1) and (2) in that statement. As seen from the proof of Theorem 5.2, the support of $\rho$ only intersects a dark gray parallelogram in the puzzle of $\mu$ along segments parallel to its edges. Note incidentally that $\rho_{\mu}=m^{\prime}$. We will show that the measure $v=p \rho_{\mu^{*}}$ satisfies the requirements of the theorem. The measure $\rho_{\mu^{*}}$ is obtained as above, by removing the white pieces and dark gray parallelograms of the puzzle of $\mu$.

Recall first that the measure $\rho$ is homologous to $m^{\prime}$, and homologous sides have equal densities. It follows that the supports of $(p \rho)^{*}$ and $\left(\mathrm{pm}^{\prime}\right)^{*}$ coincide. The measure $p \rho_{\mu^{*}}$ is obtained from $p \rho$ by simply decreasing, possibly to 0 , the length of the sides of the various segments in the support of $p \rho$. It follows that $v^{*}$ is obtained from $(p \rho)^{*}$ by decreasing, possibly to 0 , the densities of some edges. Thus assertion (1) is verified.

To verify the remaining assertions, we examine more closely the process which generates the measure $v$. Consider a lattice point in the support of $\mu$, and examine 
the inflation applied to the six small edges adjacent to this vertex.
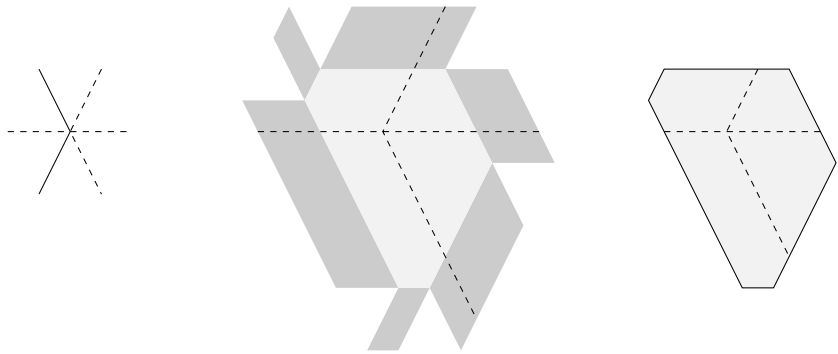

The support of $m^{\prime}$ will contain at most four of these six edges, and these are indicated by dotted lines. The support of $\rho$ is also indicated by dotted lines in the inflation. Next, to obtain the support of $\mu^{*}$, we replace the dark gray parallelograms by solid edges in the boundary of the light gray puzzle piece.

Consider now the puzzle of the measure $p \rho$ which covers a triangle with sides

$$
r+\omega(\mu)+p \omega(\rho)=r+\omega(\mu)+p \omega\left(m^{\prime}\right)=r+\omega(m),
$$

which is also the size of the puzzle of $m$. We want to preserve the already existing colors, so that we introduce three new ones. The parts of $\operatorname{supp}(p \rho)$ contained in white puzzle pieces, or contained in dark gray parallelograms and parallel to their white sides, inflate to darker gray parallelograms. The parts contained in the light gray puzzle pieces, or in dark gray parallelograms and parallel to the light gray sides, inflate to black parallelograms. Finally, the puzzle pieces arising from branch points of $p \rho$ are medium gray. Thus, the darker gray parallelograms correspond to the original edges in the support of $m^{\prime}$, translated to the puzzle of $\mu$, while the black parallelograms are obtained from the edges added in order to fill the gaps in the support of $\rho$. The following illustration represents the generic case, with the highest possible number of nonzero densities. When some densities are zero, the figure degenerates in various ways. For instance, the lattice point might not be a branch point of $\mu$ (in which case there is no light gray piece), or one of the edges may be in $\operatorname{supp}\left(m^{\prime}\right) \backslash \operatorname{supp}(\mu)$ (in which case the support of $\rho$ intersects dark gray parallelograms only along their edges).
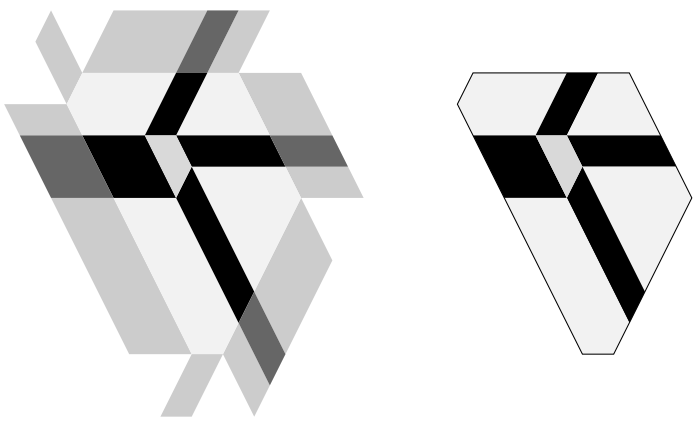
This picture of the puzzle of $p \rho$ occupies the same triangle as the puzzle of $m$. In fact, one can identify the various pieces in the puzzle of $m$ as follows. The union of the dark and darker gray parallelograms form precisely the inflated pieces of the support of $m$, while the white pieces are translates of the connected components of $\triangle_{r} \backslash \operatorname{supp}(m)$. The light gray, medium gray, and black areas reconstitute the pieces in the puzzle of $m$ corresponding to branch points. Therefore, replacing the dark and darker gray parallelograms with solid lines (which are assigned density equal to the length of the white sides of the parallelograms) and deleting the white puzzle pieces we obtain the dual $\mathrm{m}^{*}$. Preserving the color scheme adopted above for the puzzle of $p \rho$, the picture also displays the puzzle of $v$. Deleting now the black parallelograms and the medium gray puzzle pieces we obtain the measure $\mu^{*}$, while deleting the light gray pieces and the black parallelograms we obtain the measure $\left(m^{*}\right)_{v^{*}}$ with support contained in that of $v^{*}$.
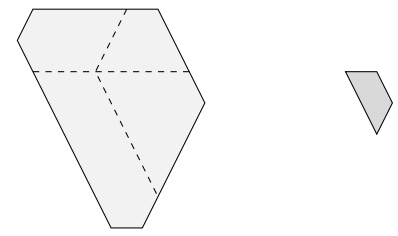

The theorem follows.

The preceding result yields a proof of Corollary 4.3 based on the factorization of Littlewood-Richardson coefficients. With the notation of the theorem, we have

$$
c_{m}=c_{m^{*}}=c_{\left(m^{*}\right)_{\nu}} c_{\left(m^{*}\right)_{\nu^{*}}}=c_{\mu^{*}}=c_{\mu},
$$

where we use the rigidity of $\left(m^{*}\right)_{v^{*}}$ in the third equality.

Remark 5.4 implies that Theorem 7.3 is true in the more general situation when $m^{\prime}$ can be written as $m^{\prime}=p_{1} m_{1}+p_{2} m_{2}+\cdots+p_{N} m_{N}$, where each $m_{j}$ is a tree measure such that $\Sigma_{m_{j}}(\mu)=0$. This produces a saturated Horn inequality for $m^{*}$. Consider in particular the case of a rigid measure $m$, and write it as in Corollary 4.5, that is $m=p_{1} m_{1}+p_{2} m_{2}+\cdots+p_{n} m_{n}$, where each $m_{j}$ is a rigid tree measure and $\Sigma_{m_{j}}\left(m_{i}\right)=0$ for $j<i$. In this case we obtain $n-1$ saturated, rigid, Horn inequalities for $m^{*}$ by applying this observation to measures of the form $m^{\prime}=a_{1} m_{1}+\cdots+a_{N} m_{N}$ with $N<n$. There may be more such inequalities because the order induced by $\prec_{0}$ on the set $\left\{m_{1}, m_{2}, \ldots, m_{n}\right\}$ is not generally total.

Corollary 7.4 Consider a rigid measure $m \in \mathcal{M}_{r}$ which assigns integer densities to all edges. If $m^{*}$ saturates no Horn inequality, then $m$ is extremal. Conversely, if $m$ is a tree measure, then $m^{*}$ saturates no Horn inequalities.

Proof The first assertion follows from the discussion above. Assume therefore that $m$ is a tree measure and $m^{*}$ saturates the Horn inequality determined by the puzzle of some measure $v$. Then the measures $\left(m^{*}\right)_{v}$ and $\left(m^{*}\right)_{v^{*}}$ assign integer densities to all edges, and $\left(\left(m^{*}\right)_{v}\right)^{*}+\left(\left(m^{*}\right)_{v^{*}}\right)^{*}=m$. Thus $m$ is the sum of two nonzero measures which assign integer densities to all edges, and this is not possible because $m$ assigns unit density to its root edges. 
Simple examples show that $(2 m)^{*}$ may saturate some Horn inequality when $m$ is a rigid tree measure.

\section{An arboretum of rigid tree measures}

The reduction procedure described in Sect. 6 requires, given a measure $m \in \mathcal{M}_{r}$, finding a rigid extremal measure $m^{\prime} \in \mathcal{M}_{r}$ such that $\Sigma_{m^{\prime}}(m)<0$. We have seen that such measures $m^{\prime}$ have the support contained in the support of $m$. Unfortunately, it is easy to construct measures $m$ with very large support. For instance, let $\mu_{1}, \mu_{2}, \ldots, \mu_{N}$ be an enumeration of all the rigid tree measures in $\mathcal{M}_{r}$, and let $c_{1}, c_{2}, \ldots, c_{N}$ be positive integers. Then the support of the measure $m=\sum_{i=1}^{N} c_{i} \mu_{i}$ contains every small edge in $\triangle_{r}$. Moreover,

$$
\Sigma_{\mu_{j}}(m)=-c_{j}+\sum_{i \neq j} c_{i} \Sigma_{\mu_{j}}\left(\mu_{i}\right), \quad j=1,2, \ldots, N .
$$

Thus the choice $m^{\prime}=\mu_{j}$ is possible provided that $c_{j}$ is sufficiently large. In other words, any rigid tree measure might serve as $m^{\prime}$ in this procedure, and an exhaustive application of our reductions would require a complete catalogue of rigid tree measures in $\mathcal{M}_{r}$. We are not aware of any algorithmic construction of such a catalogue, and we are in possession of a complete list only for $r \leq 6$. In this section we have a more modest goal: we use Theorem 4.2 to describe those rigid measures which have precisely three nonzero exit densities on each side of $\triangle_{r}$. Assume thus that the rigid tree measure $m \in \mathcal{M}_{r}$ has weight $\omega$, and nonzero densities $\alpha, \alpha^{\prime}, \alpha^{\prime \prime}$ in the NW direction, $\beta, \beta^{\prime}, \beta^{\prime \prime}$ in the $\mathrm{SW}$ direction, and $\gamma, \gamma^{\prime}, \gamma^{\prime \prime}$ in the E direction. These integers must satisfy

$$
\alpha+\alpha^{\prime}+\alpha^{\prime \prime}=\beta+\beta^{\prime}+\beta^{\prime \prime}=\gamma+\gamma^{\prime}+\gamma^{\prime \prime}=\omega,
$$

and

$$
\alpha^{2}+\alpha^{\prime 2}+\alpha^{\prime \prime 2}+\beta^{2}+\beta^{\prime 2}+\beta^{\prime \prime 2}+\gamma^{2}+\gamma^{\prime 2}+\gamma^{\prime \prime 2}=\omega^{2}+2
$$

by Theorem 4.2(3). Assume first that $\omega=3 k+1$ for some integer $k \geq 1$. The smallest value allowed by (8.1) for the sum

$$
\alpha^{2}+\alpha^{\prime 2}+\alpha^{\prime \prime 2}+\beta^{2}+\beta^{\prime 2}+\beta^{\prime \prime 2}+\gamma^{2}+\gamma^{\prime 2}+\gamma^{\prime \prime 2}
$$

is achieved when the weights on each side are $k, k$ and $k+1$, and that value is precisely $\omega^{2}+2$. Thus (8.2) implies that the weights on each side have precisely these values (in some order). Similarly, when $\omega=3 k+2$, the densities on each side must be $k, k+1$ and $k+1$. When $\omega=3(k+1)$, relation (8.2) implies

$$
\alpha^{2}+\alpha^{\prime 2}+\alpha^{\prime \prime 2}+\beta^{2}+\beta^{\prime 2}+\beta^{\prime \prime 2}+\gamma^{2}+\gamma^{\prime 2}+\gamma^{\prime \prime 2} \geq 9(k+1)^{2}=\omega^{2},
$$

with equality achieved only when all the exit densities are equal to $k+1$. It follows easily from (8.2) that on two sides the exit densities will all be equal to $k+1$, while 
on the remaining side they must be $k, k+1, k+2$. We will now produce actual examples of rigid tree measures with three nonzero exit densities in each direction, and with all possible values of $\omega$. A first series of examples is described in the following figure.
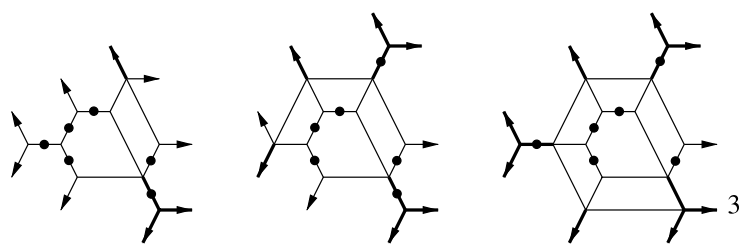

The thinner edges have density one, and the thicker ones have density two, except for one exit density which is equal to three, as labeled. Other such measures can be obtained by applying $120^{\circ}$ rotations to these measures, or symmetries about a horizontal line. Another way to obtain new measures is to change the lengths of the edges indicated by a dot. These lengths can be chosen arbitrarily; here is an example of this procedure applied to the second measure above.

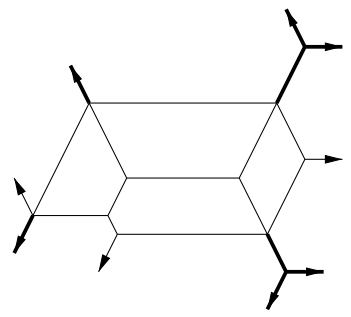

The three measures above provide examples with $\omega=3 k+1,3 k+2$ and $3(k+1)$ when $k=1$. For larger values of $k$ one must continue the spiral pattern. A second series of examples is illustrated below.
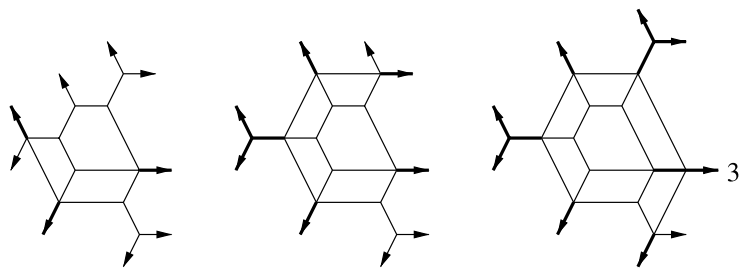

As in the first series of examples, these measures can be rotated by multiples of $120^{\circ}$, and reflected in a horizontal line. Their shapes can also be changed by modifying arbitrarily the lengths of six of the edges. Again, the spiral can be continued to yield examples with weights $3 k+1,3 k+2$ and $3(k+1)$ for all integers $k \geq 1$. 
A third series of examples is illustrated next.
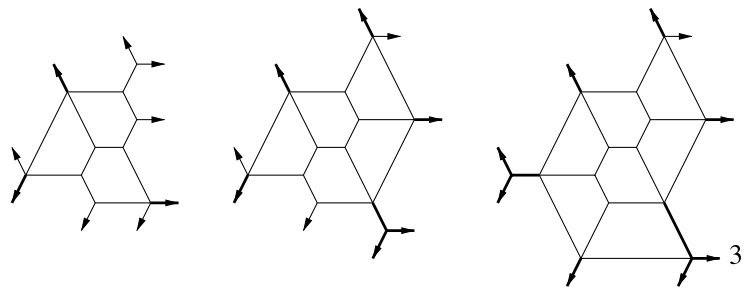

Note that this series has two spiral arms. To obtain measures with higher weight one proceeds by alternately increasing each spiral by $1 / 3$ of a complete turn.

When $\omega(m)=3 k+1$ there is one more series of measures which have greater symmetry. The first two in the series are pictured below.
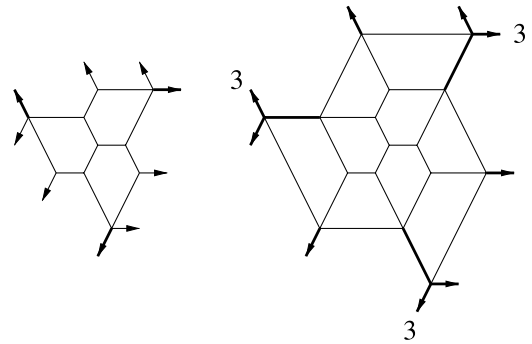

These measures are invariant under $120^{\circ}$ rotations, but not under reflection relative to a horizontal line.

A similar series is available for $\omega=3 k+2$.
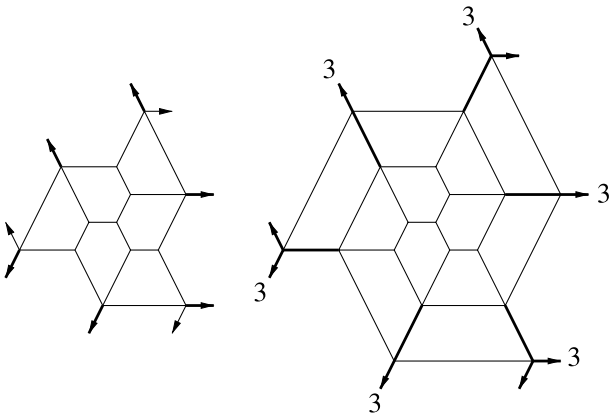

Some of these examples have versions for $k=0$, though in that case there will be fewer than three nonzero exit densities in some direction. Using duality of measures, it can be shown that the measures described above (along with their rotations, reflections and stretched versions) are the only measures with exactly three nonzero exit densities in each direction. Thus, for instance, there are no rigid tree measures whose exit densities are (in counterclockwise order, starting with $\alpha)(k+1, k+1, k)$, $(k+1, k, k+1)$ and $(k, k+1, k+1)$ or $(k+1, k, k),(k, k, k+1)$ and $(k, k+1, k)$. 


\section{References}

1. Bercovici, H., Collins, B., Dykema, K., Li, W.S., Timotin, D.: Intersections of Schubert varieties and eigenvalue inequalities in an arbitrary finite factor. J. Funct. Anal. 258, 1579-1627 (2010)

2. Buch, A.S.: The saturation conjecture (after A. Knutson and T. Tao). With an appendix by William Fulton. Enseign. Math. (2) 46(1-2), 43-60 (2000)

3. Collins, B., Dykema, K.: On a reduction procedure for Horn inequalities in finite von Neumann algebras. Oper. Matrices 3, 1-40 (2009)

4. Danilov, V.I., Koshevoy, G.A.: Discrete convexity and Hermitian matrices. Tr. Mat. Inst. Steklova 241, 68-89 (2003); translation in Proc. Steklov Inst. Math. 241, 58-78 (2003)

5. Derksen, H., Weyman, J.: The combinatorics of quiver representations, preprint, 2006, arXiv:math/ 0608288

6. Fulton, W.: Young Tableaux. Cambridge University Press, Cambridge (1997)

7. King, R.C., Tollu, C., Christophe, F.: Factorisation of Littlewood-Richardson coefficients. J. Combin. Theory Ser. A 116, 314-333 (2009)

8. Knutson, A., Tao, T., Woodward, C.: The honeycomb model of $\mathrm{GL}_{n}(\mathbb{C})$ tensor products. II. Puzzles determine facets of the Littlewood-Richardson cone. J. Am. Math. Soc. 17, 19-48 (2004)

9. Thompson, R.C., Therianos, S.: On a construction of B.P. Zwahlen. Linear Multilinear Algebra 1, 309-325 (1973/74) 